\title{
Superconformal Chern-Simons theories from del Pezzo geometries
}

\author{
Sanefumi Moriyama, ${ }^{a, b}$ Tomoki Nosaka $^{c}$ and Katsuya Yano ${ }^{a}$ \\ ${ }^{a}$ Department of Physics, Graduate School of Science, Osaka City University, \\ Sumiyoshi-ku, Osaka 558-8585, Japan \\ ${ }^{b}$ Osaka City University Advanced Mathematical Institute (OCAMI), \\ Sumiyoshi-ku, Osaka 558-8585, Japan \\ ${ }^{c}$ Korea Institute for Advanced Study, \\ Dongdaemun-gu, Seoul 02455, Korea \\ E-mail: moriyama@sci.osaka-cu.ac.jp, nosaka@yukawa.kyoto-u.ac.jp, \\ yanok@sci.osaka-cu.ac.jp
}

ABSTRACT: We present an explicit expression for the grand potential of the $\mathrm{U}(N)^{3}$ superconformal Chern-Simons theory with the Chern-Simons levels being $(k, 0,-k)$. From the viewpoint of the Newton polygon, it is expected that the grand potential is given by the free energy of the topological string theory on the local $D_{5}$ del Pezzo geometry, though the explicit identification was a puzzle for years. We show how the expectation is realized explicitly. As a bonus, we can also study the $\mathbb{Z}_{2}$ orbifold of this theory and find the grand potential is now given in terms of the local $E_{7}$ del Pezzo geometry.

KEYwords: Chern-Simons Theories, Matrix Models, Nonperturbative Effects, Topological Strings

ArXiv EPrint: 1707.02420 


\section{Contents}

1 Introduction 1

$2(2,1)$ model $\quad 4$

3 Observations $\quad 7$

$\begin{array}{lll}3.1 & \text { Worldsheet instanton relation } & 7\end{array}$

3.2 Multi-covering structure for membrane instantons 8

$\begin{array}{ll}3.3 \text { Group-theoretical viewpoint } & 9\end{array}$

4 Topological string $\quad 10$

4.1 Kähler parameters 11

$\begin{array}{lll}4.2 & \text { BPS indices } & 13\end{array}$

$\begin{array}{lll}4.3 \text { Characters } & 15\end{array}$

$\begin{array}{ll}4.4 \text { Higher degrees } & 16\end{array}$

5 Rank-deformed $(2,2)$ model from characters $\quad 18$

6 Orbifold $(2,1)$ model $\quad 20$

6.1 Instantons 20

$\begin{array}{lll}6.2 \text { Characters } & 22\end{array}$

$\begin{array}{lll}7 & \text { Discussions } & 25\end{array}$

A Data for $(2,1) /(2,2)$ model and $D_{5} \quad 26$

A.1 Instanton coefficients for $(2,1)$ model 26

A.2 Decomposition of so(10) representations 28

A.3 BPS indices for so(10) representations 30

A.4 Characters for rank-deformed $(2,2)$ model 32

B Data for $(2,1,2,1)$ model and $E_{7} \quad 33$

B.1 Instanton coefficients for $(2,1,2,1)$ model 33

B.2 Decomposition of $E_{7}$ representations 35

\section{Introduction}

M-theory, though it was proposed to unify all of the five perturbative string theories, has been a mysterious theory for a long time. Recently this theory was demystified largely partially due to the discovery of the worldvolume theory of the fundamental M2-branes. Namely, it was proposed [1-3] that the worldvolume theory of $\min \left(N_{1}, N_{2}\right)$ M2-branes and 
$\left|N_{2}-N_{1}\right|$ fractional M2-branes on the target space geometry $\mathbb{C}^{4} / \mathbb{Z}_{k}$ is described by the $\mathcal{N}=6$ superconformal Chern-Simons theory with the gauge group $\mathrm{U}\left(N_{1}\right)_{k} \times \mathrm{U}\left(N_{2}\right)_{-k}$ and two pairs of bifundamental matters where the subscripts $(k,-k)$ denote the Chern-Simons levels.

Due to the localization techniques $[4,5]$, the infinite-dimensional path integral in defining the partition function of the ABJM theory on $S^{3}$ is reduced to a finite-dimensional matrix integration. It is convenient to consider the reduced grand potential ${ }^{1} J(\mu)[6]$ for the partition function by regarding the rank $N=\min \left(N_{1}, N_{2}\right)$ as the number of particles and introducing the dual chemical potential $\mu$ [7]. Then, it was known $[8]^{2}$ that, if we further redefine the effective chemical potential $\mu_{\text {eff }}$ appropriately [16], aside from the perturbative part of the reduced grand potential given by a cubic polynomial of the effective chemical potential $[7,9,12]$, the non-perturbative part is separated into that of pure worldsheet instantons $[9,17]$ and that of pure membrane instantons [11], $J^{\mathrm{np}}\left(\mu_{\mathrm{eff}}\right)=J^{\mathrm{WS}}\left(\mu_{\mathrm{eff}}\right)+J^{\mathrm{MB}}\left(\mu_{\mathrm{eff}}\right)$. The worldsheet instanton $J^{\mathrm{WS}}\left(\mu_{\text {eff }}\right)$ takes the form of the free energy of the topological string theory, while the membrane instanton $J^{\mathrm{MB}}\left(\mu_{\mathrm{eff}}\right)$ takes the form of the derivative of the free energy of the refined topological string theory in the Nekrasov-Shatashvili limit $\left(s_{\mathrm{L} / \mathrm{R}}=2 j_{\mathrm{L} / \mathrm{R}}+1\right)$

$$
\begin{aligned}
J^{\mathrm{WS}}\left(\mu_{\mathrm{eff}}\right) & =\sum_{j_{\mathrm{L}}, j_{\mathrm{R}}} \sum_{\boldsymbol{d}} N_{j_{\mathrm{L}}, j_{\mathrm{R}}}^{\boldsymbol{d}} \sum_{n=1}^{\infty} \frac{(-1)^{\left(s_{\mathrm{L}}+s_{\mathrm{R}}-1\right) n} s_{\mathrm{R}} \sin 2 \pi g_{\mathrm{s}} n s_{\mathrm{L}}}{n\left(2 \sin \pi g_{\mathrm{s}} n\right)^{2} \sin 2 \pi g_{\mathrm{s}} n} e^{-n \boldsymbol{d} \cdot \boldsymbol{T}}, \\
J^{\mathrm{MB}}\left(\mu_{\mathrm{eff}}\right) & =\sum_{j_{\mathrm{L}}, j_{\mathrm{R}}} \sum_{\boldsymbol{d}} N_{j_{\mathrm{L}}, j_{\mathrm{R}}}^{\boldsymbol{d}} \sum_{n=1}^{\infty} \frac{\partial}{\partial g_{\mathrm{s}}}\left[g_{\mathrm{s}} \frac{-\sin \frac{\pi n}{g_{\mathrm{s}}} s_{\mathrm{L}} \sin \frac{\pi n}{g_{\mathrm{s}}} s_{\mathrm{R}}}{4 \pi n^{2}\left(\sin \frac{\pi n}{g_{\mathrm{s}}}\right)^{3}} e^{-n \frac{\boldsymbol{d} \cdot \boldsymbol{T}}{g_{\mathrm{s}}}}\right] .
\end{aligned}
$$

Here the two Kähler parameters and the string coupling constant are identified as

$$
T^{ \pm}=\frac{4 \mu_{\mathrm{eff}}}{k} \pm \pi i\left(1-\frac{2 M}{k}\right), \quad g_{\mathrm{s}}=\frac{2}{k},
$$

with $M=N_{2}-N_{1}$ and $N_{j_{\mathrm{L}}, j_{\mathrm{R}}}^{d}$ is the BPS indices of the local $\mathbb{P}^{1} \times \mathbb{P}^{1}$ geometry (see $[18,19]$ for reviews). The appearance of the topological string theory and the local $\mathbb{P}^{1} \times \mathbb{P}^{1}$ geometry may look surprising at first sight. This is partially motivated by the Fermi gas formalism [7], which rewrites the partition function of the ABJM theory into that of a non-interacting Fermi gas system. The spectral operator of this system is given by $e^{\widehat{H}}=\left(2 \cosh \frac{\widehat{q}}{2}\right)\left(2 \cosh \frac{\widehat{p}}{2}\right)$ where $\widehat{q}$ and $\widehat{p}$ are the canonical position/momentum operators. Then, it was observed [7] that the Newton polygon of the classical spectral curve $\sum_{m, n} e^{m q+n p}=e^{E}$ with $m, n= \pm \frac{1}{2}$ is nothing but that of the $\mathbb{P}^{1} \times \mathbb{P}^{1}$ geometry under the change of variables.

After establishing the results for the M2-branes on the background with large supersymmetry, it is interesting to explore more general backgrounds. Namely, we can naturally ask what happens when we consider other superconformal Chern-Simons theories, which are natural generalizations of the ABJM theory. Especially, we are interested in whether the non-perturbative part of the reduced grand potential of those superconformal ChernSimons theories falls into the same expression (1.1), or if not, what the generalization

\footnotetext{
${ }^{1}$ See (2.3) later for the definition of the reduced grand potential.

${ }^{2}$ See also $[6,7,9-16]$ for earlier works leading to this result.
} 
of (1.1) is. Interestingly, in [20] it was conjectured that the reduced grand potential of a large class of the spectral determinants falls into the same expression as (1.1), where the geometry is read off from the classical spectral curve as in the case of the ABJM theory.

The investigation of the grand potential of general superconformal Chern-Simons theories starts from a special class enjoying the supersymmetry $\mathcal{N}=4$. It was found [21-25] that for the circular quiver of unitary gauge groups the superconformal Chern-Simons theory enjoys the supersymmetry enhancement of $\mathcal{N}=4$ if the Chern-Simons levels satisfy $k_{a}=(k / 2)\left(s_{a}-s_{a-1}\right)$ with $s_{a}= \pm 1$.

One of the simplest models [26] among the $\mathcal{N}=4$ superconformal Chern-Simons theories is the theory with the gauge group $\mathrm{U}(N)_{k} \times \mathrm{U}(N)_{0} \times \mathrm{U}(N)_{-k} \times \mathrm{U}(N)_{0}$, which is dubbed $(2,2)$ model from the number of \pm 1 appearing continuously in $\left\{s_{a}\right\}=\{+1,+1,-1,-1\}$. In fact, it was observed [26] that the non-perturbative part of the grand potential has the structure of (1.1) with $g_{s}=1 / k$ and a particular choice of Kähler parameters T. Moreover, the diagonal Gopakumar-Vafa invariants, special combinations of the BPS indices, of the $(2,2)$ model match with those of the local $D_{5}$ del Pezzo geometry. This is indeed natural from the viewpoint of the Newton polygon since the spectral curve of the $(2,2)$ model is $\sum_{m, n} e^{m q+n p}=e^{E}$ with $m, n=0, \pm 1$.

Due to the complexity with large degrees of freedom, it was difficult to study this generalization carefully. Very recently, from the improvements in the Fermi gas formalism, ${ }^{3}$ we were able to revisit the $(2,2)$ model by considering the rank deformations [36] and found that the reduced grand potential of the rank deformed $(2,2)$ model still falls into the same non-perturbative expression (1.1) with the total BPS indices listed in [37] split in a very non-trivial way. We also studied the rank deformations of the $\mathbb{Z}_{2}$ orbifold ${ }^{4}$ of the ABJM theory, or the $(1,1,1,1)$ model with $\left\{s_{a}\right\}=\{+1,-1,+1,-1\}$, which are connected to the $(2,2)$ model at the edge of the rank deformations through the Hanany-Witten duality [38]. We found that the free energy of the topological string theory (1.1) unifies the moduli space of the rank deformations of these two dual models with the six Kähler parameters of the local $D_{5}$ del Pezzo geometry. From this unified viewpoint, the worldsheet instanton exponent $e^{-\frac{2 \mu_{\text {eff }}}{k}}$ of the $(1,1,1,1)$ model is realized by a non-trivial cancellation in the worldsheet instantons whose exponent is generically $e^{-\frac{\mu_{\text {eff }}}{k}}$.

Another interesting model is the $(2,1)$ model with the gauge group $\mathrm{U}(N)_{k} \times \mathrm{U}(N)_{0} \times \mathrm{U}(N)_{-k}$ whose levels are specified by $\left\{s_{a}\right\}=\{+1,+1,-1\}$. Although the study of this model dates back to [39], it was, however, difficult to find the general structure for a long time. In this paper, we shall present a complete description of the $(2,1)$ model (without rank deformations). We have found that the description of the $(2,1)$ model falls into the same expression as (1.1) if we choose the Kähler parameters and the BPS indices appropriately, though it looks quite different at first sight.

The study of this model is interesting also from the viewpoint of the Newton polygon. Though the spectral curve of the $(2,1)$ model is $\sum_{m, n} e^{m q+n p}=e^{E}$ with $m=0, \pm 1$,

\footnotetext{
${ }^{3}$ See [27-35] for related improvements in the Fermi gas formalism.

${ }^{4}$ The physical interpretation of the repetition of the spectral operator is the orbifold in the target space of the M2-branes [22, 23]. This should not be confused with the orbifold in the background geometry of the topological string theory.
} 
$n= \pm \frac{1}{2}$, we cannot consistently truncate to these points in the Newton polygon. In fact, after rescaling $p / 2 \rightarrow p$, the Newton polygon is indistinguishable as a convex hull from that for the $(2,2)$ model. So our main task in this paper is to identify how the $D_{5}$ del Pezzo geometry appears in the $(2,1)$ model. After observing that the instanton expression of the $(2,1)$ model keeps many BPS indices of the local $D_{5}$ del Pezzo geometry as mementos, we construct a framework so that these mementos can be utilized to describe the model correctly.

Considering the rather long analysis of the $(2,1)$ model starting from [39], our resulting statement is surprisingly short. The reduced grand potential of the $(2,1)$ model is given by the same expression of topological strings (1.1) with the four Kähler parameters

$$
T_{\uparrow}^{ \pm}=\frac{2 \mu_{\mathrm{eff}}}{k} \pm \pi i\left(1+\frac{1}{k}\right), \quad T_{\downarrow}^{ \pm}=\frac{2 \mu_{\mathrm{eff}}}{k} \pm \pi i\left(-1+\frac{1}{k}\right) .
$$

The BPS indices are obtained by identifying those of the local $D_{5}$ del Pezzo geometry as the representations of the original algebra so(10) and decomposing the representations to the subalgebra $\mathrm{so}(6) \times \mathrm{u}(1) \times \mathrm{u}(1)$ where the two $\mathrm{u}(1)$ charges are identified respectively as the two degree differences of \pm and $\uparrow \downarrow$.

As a bonus of our study, we can also study the $\mathbb{Z}_{2}$ orbifold of the $(2,1)$ model, that is, the $(2,1,2,1)$ model with $\left\{s_{a}\right\}=\{+1,+1,-1,+1,+1,-1\}$. We have identified the reduced grand potential of the $(2,1,2,1)$ model with the topological string description (1.1) with the BPS indices being those of the local $E_{7}$ del Pezzo geometry. This is motivated by a suggestive expression of the Newton polygon of the $E_{7}$ del Pezzo geometry in [40].

The organization of this paper is as follows. In section 2 we first review the known results of the $(2,1)$ model. After acquiring some clues from the observations on the relation to the rank-deformed $(2,2)$ model with the gauge group $\mathrm{U}(N)_{k} \times \mathrm{U}(N+M)_{0} \times \mathrm{U}(N+2 M)_{-k} \times \mathrm{U}(N+M)_{0}$ and on the group-theoretical viewpoint for the $(2,2)$ model in section 3 , in section 4 we present carefully how the reduced grand potential is described with the free energy of topological strings. In section 5 we shortly revisit the two-parameter rank deformation of the $(2,2)$ model $\mathrm{U}\left(N+M_{\mathrm{II}}\right)_{k} \times \mathrm{U}\left(N+M_{\mathrm{I}}\right)_{0} \times \mathrm{U}\left(N+2 M_{\mathrm{I}}+M_{\mathrm{II}}\right)_{-k} \times \mathrm{U}\left(N+M_{\mathrm{I}}\right)_{0}$ studied in [36] by expressing the reduced grand potential in a more economical language of characters. In section 6 we turn to the $(2,1,2,1)$ model and describe the reduced grand potential of this model using the language of characters. Finally we conclude with some discussions.

In appendix A we summarize the instanton coefficients and the group-theoretical data which are necessary in order to check the relation between the representation theory for so(10) and the instanton coefficients of the $(2,1)$ model and the rank deformed $(2,2)$ model. Appendix B is the collection of the instanton coefficients of the $(2,1,2,1)$ model and the group-theoretical data for $E_{7}$ and so(12) relevant to our proposal.

\section{$2(2,1)$ model}

In this section we review the result for the $(2,1)$ model $[26,39]$ shortly. The infinitedimensional path integral in defining the partition function of the $(2,1)$ model is reduced 
to a finite-dimensional matrix integration [5]

$$
\begin{aligned}
& Z(N) \\
& =\int \frac{D^{N} \mu}{N !} \frac{D^{N} \lambda}{N !} \frac{D^{N} \nu}{N !} \frac{\prod_{m<m^{\prime}}^{N}\left(2 \sinh \frac{\mu_{m}-\mu_{m^{\prime}}}{2}\right)^{2} \prod_{l<l^{\prime}}^{N}\left(2 \sinh \frac{\lambda_{l}-\lambda_{l^{\prime}}}{2}\right)^{2} \prod_{n<n^{\prime}}^{N}\left(2 \sinh \frac{\nu_{n}-\nu_{n^{\prime}}}{2}\right)^{2}}{\prod_{m, l}^{N} 2 \cosh \frac{\mu_{m}-\lambda_{l}}{2} \prod_{l, n}^{N} 2 \cosh \frac{\lambda_{l}-\nu_{n}}{2} \prod_{n, m}^{N} 2 \cosh \frac{\nu_{n}-\mu_{m}}{2}},
\end{aligned}
$$

with the integrations

$$
D \mu=\frac{d \mu}{2 \pi} e^{\frac{i k}{4 \pi} \mu^{2}}, \quad D \lambda=\frac{d \lambda}{2 \pi}, \quad D \nu=\frac{d \nu}{2 \pi} e^{-\frac{i k}{4 \pi} \nu^{2}} .
$$

It was found that the reduced grand potential of the $(2,1)$ model defined as ${ }^{5}$

$$
\sum_{n=-\infty}^{\infty} e^{J(\mu+2 \pi i n)}=\sum_{N=0}^{\infty} e^{N \mu} Z(N),
$$

by introducing the chemical potential $\mu$ dual to the rank $N$, is given separately as the summation of the worldsheet instanton part and the membrane instanton part

$$
J(\mu)=J^{\text {pert }}\left(\mu_{\mathrm{eff}}\right)+J^{\mathrm{np}}\left(\mu_{\mathrm{eff}}\right), \quad J^{\mathrm{np}}\left(\mu_{\mathrm{eff}}\right)=J^{\mathrm{WS}}\left(\mu_{\mathrm{eff}}\right)+J^{\mathrm{MB}}\left(\mu_{\mathrm{eff}}\right),
$$

aside from the perturbative part,

$$
J^{\text {pert }}\left(\mu_{\mathrm{eff}}\right)=\frac{C}{3} \mu_{\mathrm{eff}}^{3}+B \mu_{\mathrm{eff}}+A, \quad C=\frac{1}{\pi^{2} k}, \quad B=-\frac{1}{12 k}+\frac{k}{12},
$$

with $A$ given in [39], if we reexpress with the effective chemical potential $\mu_{\text {eff }}$ suitably. For integral $k, \mu_{\mathrm{eff}}$ is given by

$$
\mu_{\mathrm{eff}}= \begin{cases}\mu-2 e^{-2 \mu}{ }_{4} F_{3}\left(1,1, \frac{3}{2}, \frac{3}{2} ; 2,2,2 ; 16 e^{-2 \mu}\right), & \text { for odd } k, \\ \mu-6 e^{-2 \mu}{ }_{4} F_{3}\left(1,1, \frac{7}{4}, \frac{5}{4} ; 2,2,2 ; 64 e^{-2 \mu}\right), & \text { for even } k,\end{cases}
$$

where the first few non-perturbative terms are extrapolated into real functions of $k$ using the WKB expansion [26].

The worldsheet instantons are given by

$$
J^{\mathrm{WS}}\left(\mu_{\mathrm{eff}}\right)=\sum_{m=1}^{\infty} d_{m} e^{-m \frac{2 \mu_{\mathrm{eff}}}{k}} .
$$

The coefficients $d_{m}$ are determined as real functions of $k$ by the interpolation from the coefficients at integral $k$, which are found to satisfy the multi-covering structure

$$
d_{m}=\sum_{n \mid m} \frac{1}{n} \delta \frac{m}{n}\left(\frac{k}{n}\right),
$$

\footnotetext{
${ }^{5}$ See [6] for an explanation on the reason to study the reduced grand potential instead of the original grand potential, which is defined simply as $e^{J^{\text {original }}(\mu)}$ for the same right-hand side of (2.3).
} 
where the multi-covering component $\delta_{d}(k)$ takes the following form

$$
\delta_{d}(k)=\frac{\sum_{n} \delta_{d, n} \cos \frac{\pi n}{k}}{\left(2 \sin \frac{2 \pi}{k}\right)^{2}}
$$

with a finite number of non-vanishing integral coefficients $\delta_{d, n}$ at each degree. The first several components $\delta_{d}(k)$ are summarized in appendix A.1.

The membrane instantons are given by the general form

$$
J^{\mathrm{MB}}\left(\mu_{\mathrm{eff}}\right)=\widetilde{J}_{b}\left(\mu_{\mathrm{eff}}\right) \mu_{\mathrm{eff}}+\widetilde{J}_{c}\left(\mu_{\mathrm{eff}}\right), \quad \widetilde{J}_{b}\left(\mu_{\mathrm{eff}}\right)=\sum_{\ell=1}^{\infty} \widetilde{b}_{2 \ell} e^{-2 \ell \mu_{\mathrm{eff}}}, \quad \widetilde{J}_{c}\left(\mu_{\mathrm{eff}}\right)=\sum_{\ell=1}^{\infty} \widetilde{c}_{\ell} e^{-\ell \mu_{\mathrm{eff}}}
$$

where the instanton coefficients of odd instantons $\widetilde{c}_{2 \ell-1}$ are constants in $\mu_{\text {eff }}$, while those of even instantons are the standard linear polynomials in $\mu_{\text {eff }}$ with $\widetilde{b}_{2 \ell} \mu_{\text {eff }}+\widetilde{c}_{2 \ell}$ satisfying the derivative relation

$$
\widetilde{c}_{2 \ell}=-k^{2} \frac{d}{d k} \frac{\widetilde{b}_{2 \ell}}{2 \ell k}
$$

The first several coefficients were investigated from the WKB expansion up to $\mathcal{O}\left(k^{9}\right)$ in $[26,39]$. The coefficients of the odd instantons can be expressed in the following simple multi-covering structure

$$
\widetilde{c}_{2 \ell-1}=\sum_{n \mid 2 \ell-1} \frac{(-1)^{\frac{n-1}{2}}}{n} \gamma_{\frac{2 \ell-1}{n}}(n k)
$$

or explicitly

$$
\widetilde{c}_{1}=\gamma_{1}(k), \quad \widetilde{c}_{3}=-\frac{1}{3} \gamma_{1}(3 k)+\gamma_{3}(k), \quad \widetilde{c}_{5}=\frac{1}{5} \gamma_{1}(5 k)+\gamma_{5}(k), \quad \cdots,
$$

where $\gamma_{d}(k)$ takes the following form

$$
\gamma_{d}(k)=-\frac{\sum_{n} \gamma_{d, n} \sin \pi n k}{\sin ^{2} \frac{\pi k}{2}}
$$

with a finite number of positive integral coefficients $\gamma_{d, n}$ at each degree. Once we accept this multi-covering structure and utilize the WKB expansion of surprisingly high order $\mathcal{O}\left(k^{29}\right)$ [41] obtained with the derivative formalism of [42], we can further determine $\gamma_{d}(k)$ of higher degree $d$. The explicit expressions of the functions $\gamma_{d}(k)$ are listed in appendix A.1.

The multi-covering structure for the even instantons was not clearly understood. Nevertheless, we achieved to determine the first few coefficients without recognizing the multicovering structure

$$
\widetilde{b}_{2}=\frac{8+11 \cos \pi k+8 \cos 2 \pi k+\cos 3 \pi k}{\pi \sin 2 \pi k},
$$

$\widetilde{b}_{4}=\frac{136+256 \cos \pi k+255 \cos 2 \pi k+192 \cos 3 \pi k+136 \cos 4 \pi k+64 \cos 5 \pi k+21 \cos 6 \pi k}{2 \pi \sin 4 \pi k}$,

from the ansatz

$$
\widetilde{b}_{2 \ell}=\frac{\sum_{n} \widetilde{b}_{2 \ell, n} \cos \pi n k}{\ell \pi \sin 2 \pi \ell k}
$$


with a finite number of non-vanishing integers $\widetilde{b}_{2 \ell, n}$. With the abundant WKB data [41] we could further determine higher instanton coefficients $\widetilde{b}_{2 \ell}$. Before going on to the higher instantons, however, let us provide several new observations which are essential to reveal the whole structure of the instanton coefficients.

\section{Observations}

In this section we shall make several observations for the non-perturbative part of the $(2,1)$ model and the $(2,2)$ model, which are helpful later in solving the models.

\subsection{Worldsheet instanton relation}

In [26] we observed that when setting all the cosine functions in the numerators of the worldsheet instantons of the $(2,1)$ model in (A.1) to be 1 (with the replacement of $k$ by $2 k$ ) we correctly reproduce the worldsheet instantons of the $(2,2)$ model for $1 \leq d \leq 5$. This relation is not valid any more for higher instantons, though the expressions look close. We find that this observation should be replaced by the following more accurate observation.

In [36] we studied the $(2,2)$ model with rank deformations. Among others, it was found that the worldsheet instantons of the $(2,2)$ model with the rank deformation $\mathrm{U}(N)_{k} \times \mathrm{U}(N+$ $M)_{0} \times \mathrm{U}(N+2 M)_{-k} \times \mathrm{U}(N+M)_{0}$ are given by (see (3.20) in [36])

$$
\begin{aligned}
& \delta_{1}^{(2,2)}(k, M)=\frac{4 \cos \frac{M \pi}{k}}{\sin ^{2} \frac{\pi}{k}}, \quad \delta_{2}^{(2,2)}(k, M)=-\frac{4+\cos \frac{2 M \pi}{k}}{\sin ^{2} \frac{\pi}{k}}, \quad \delta_{3}^{(2,2)}(k, M)=\frac{12 \cos \frac{M \pi}{k}}{\sin ^{2} \frac{\pi}{k}}, \\
& \delta_{4}^{(2,2)}(k, M)=-\frac{32+16 \cos \frac{2 M \pi}{k}}{\sin ^{2} \frac{\pi}{k}}+5, \quad \delta_{5}^{(2,2)}(k, M)=\frac{220 \cos \frac{M \pi}{k}+20 \cos \frac{3 M \pi}{k}}{\sin ^{2} \frac{\pi}{k}}-96 \cos \frac{M \pi}{k} .
\end{aligned}
$$

Comparing these functions with the worldsheet coefficients of the $(2,1)$ model (A.1), it is interesting to observe a close relation. Namely, if we replace $k$ by $k / 2$ and set $M= \pm 1 / 2$ in (3.1), we can reproduce the worldsheet instantons of the $(2,1)$ model (A.1) correctly

$$
\delta_{d}(k)=\delta_{d}^{(2,2)}\left(\frac{k}{2}, \pm \frac{1}{2}\right)
$$

This observation explains the match in lower instantons and the mismatch in higher instantons observed in [26]. The relation observed in [26] is correctly reproduced in lower instantons if we assume the relation (3.2). Since the cosine functions in the numerator of (3.1) comes from the rank deformation, setting the cosine functions in $\delta_{d}(k)$ to be 1 amounts to changing $M= \pm 1 / 2$ to $M=0$. When we proceed to higher instantons and perform the replacement (3.2), the numerator of (3.1) contains the cosine functions with larger arguments, which cause the mismatch after being reexpanded by the denominator $\sin ^{2} \frac{\pi}{k}$.

There is an important implication from this observation. Though in [39] and [26] it was difficult to see whether the non-perturbative part fits to (1.1), with the expression of the Kähler parameters for the rank-deformed $(2,2)$ model [36]

$$
T^{ \pm}=\frac{\mu_{\mathrm{eff}}}{k} \pm \pi i\left(1-\frac{M}{k}\right)
$$


the relation (3.2) means that we can give a general expression for the worldsheet instanton if we choose the Kähler parameters and the string coupling constant schematically as

$$
T \sim \frac{2 \mu_{\mathrm{eff}}}{k} \pm \pi i \pm \frac{\pi i}{k}, \quad g_{\mathrm{s}} \sim \frac{2}{k}
$$

If we look at the membrane instanton more carefully, however, the fit to the expression (1.1) is not so trivial since the odd membrane instantons in (2.10) does not have

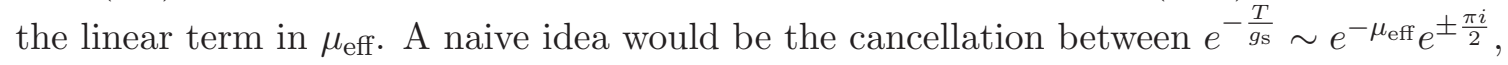
though a careful study shows that the cancellation does not work due to the extra factor in $e^{-\frac{T}{g_{\mathrm{s}}}} \sim e^{-\mu_{\mathrm{eff}}} e^{ \pm \frac{\pi k i}{2}} e^{ \pm \frac{\pi i}{2}}$. This problem, in turn, can be solved by introducing all of the four Kähler parameters in (3.4). In fact, with this setup, we shall see later in section 4.1 that the cancellation happens beautifully. The introduction of the four Kähler parameters is partially motivated by the study of the $\mathbb{Z}_{2}$ orbifold of the ABJM theory, or the $(1,1,1,1)$ model, in [36]. In relating this model to the $(2,2)$ model by changing the brane configuration, we found a non-trivial cancellation of odd instantons, which is very similar to the cancellation of the linear $\mu_{\mathrm{eff}}$ term here.

\subsection{Multi-covering structure for membrane instantons}

Once we have found the relation to the $(2,2)$ model in the worldsheet instantons, we are motivated to relate the membrane instantons of the $(2,1)$ model with those of the $(2,2)$ model as well. Interestingly, we find that the even membrane instantons (2.15) possess the following novel multi-covering structure

$$
\widetilde{b}_{2 \ell}=\sum_{n \mid 2 \ell, n \in 2 \mathbb{N}} \frac{(-1)^{\ell}}{n} \beta_{\frac{2 \ell}{n}}(n k)+\sum_{n \mid 2 \ell, n \in 2 \mathbb{N}-1} \frac{1}{n} \beta_{\frac{2 \ell}{n}}^{\prime}(n k),
$$

or explicitly

$$
\begin{aligned}
& \widetilde{b}_{2}=-\frac{1}{2} \beta_{1}(2 k)+\beta_{2}^{\prime}(k), \quad \widetilde{b}_{4}=\frac{1}{4} \beta_{1}(4 k)+\frac{1}{2} \beta_{2}(2 k)+\beta_{4}^{\prime}(k), \\
& \widetilde{b}_{6}=-\frac{1}{6} \beta_{1}(6 k)+\frac{1}{3} \beta_{2}^{\prime}(3 k)-\frac{1}{2} \beta_{3}(2 k)+\beta_{6}^{\prime}(k), \quad \cdots,
\end{aligned}
$$

where $\beta_{d}(k)$ is defined from the membrane instanton coefficient $\beta_{d}^{(2,2)}(k)$ of the $(2,2)$ model without rank deformations (see (3.15) and (3.17) in [26]) as

$$
\beta_{d}(k)=\beta_{d}^{(2,2)}\left(\frac{k}{2}\right) .
$$

Indeed, in these expansions the new component $\beta_{d}^{\prime}(k)$ at each order takes the form of

$$
\beta_{d}^{\prime}(k)=\frac{\sum_{n} \beta_{d, n} \sin \pi n k}{2 \pi \sin ^{2} \frac{\pi k}{2}},
$$

with a finite number of positive integers $\beta_{d, n}$, as in the case of the ABJM theory and the $(2,2)$ model. Once we adopt this new multi-covering structure, we can also determine the coefficients of even instantons $\beta_{d}^{\prime}(k)$ of higher degrees $d$. The explicit expressions of the 
functions $\beta_{d}^{\prime}(k)$ are summarized in appendix A.1, where the expressions of $\beta_{d}(k)$ are also recapitulated.

The above novel multi-covering structure (3.5) can be understood from the pole cancellation. As our goal is to express the instanton effects as the free energy of topological strings (1.1) where the pole cancellation occurs among the multi-covering components of each degree without mixing, it is reasonable to require the instanton coefficients to have the same substructure. The multi-covering structure (3.5) assisted with $\beta_{d}(k)$, along with (2.8) and (2.12), is very important to respect this substructure of the pole cancellation. For example let us consider the multi-covering component of degree $d=2$ in the instanton coefficient of $e^{-4 \mu_{\text {eff }}}$ at $k=2$. If we adopted $\beta_{2}^{\prime}(k)$ coming directly from $\widetilde{b}_{2}$ for the multi-covering component of $\widetilde{b}_{4}$ at degree $d=2$, the poles in the combination

$$
\frac{1}{2} \delta_{2}\left(\frac{k}{2}\right) e^{-\frac{8 \mu_{\mathrm{eff}}}{k}}+\left(\mu_{\mathrm{eff}}-k^{2} \frac{d}{d k} \frac{1}{4 k}\right) \frac{1}{2} \beta_{2}^{\prime}(2 k) e^{-4 \mu_{\mathrm{eff}}}
$$

in the limit $k \rightarrow 2$ were not cancelled any more. The reason of adopting the multi-covering structure (3.5) will be explained more carefully from the viewpoint of the free energy of topological strings (1.1) in section 4.1 .

\subsection{Group-theoretical viewpoint}

Before proceeding to the analysis, we shall explain another interesting observation. In [36] it was found that the total BPS indices identified in [37] are split due to the introduction of two Kähler parameters. We recapitulate the BPS indices discovered in [36] in table 1, though the table is rearranged in a different way. With this rearrangement it is not difficult to find the relation to the decomposition of the representations in the algebra so(10) to the subalgebra so $(8) \times \mathrm{u}(1)$. For example, the spin $\left(0, \frac{3}{2}\right)$ sector of degree 4 is reminiscent of the decomposition of the adjoint representation $\mathbf{4 5}$ and the spin $(0,2)$ sector of degree 5 is the decomposition of the representation 144

$$
\begin{aligned}
\mathbf{4 5} & \rightarrow\left(\mathbf{8}_{\mathbf{v}}\right)_{+2}+(\mathbf{2 8})_{0}+(\mathbf{1})_{0}+\left(\mathbf{8}_{\mathbf{v}}\right)_{-2} \\
\mathbf{1 4 4} & \rightarrow\left(\mathbf{8}_{\mathbf{s} / \mathbf{c}}\right)_{+3}+\left(\mathbf{5 6} \mathbf{s}_{\mathbf{s} / \mathbf{c}}\right)_{+1}+\left(\mathbf{8}_{\mathbf{s} / \mathbf{c}}\right)_{+1}+\left(\mathbf{5} \mathbf{6}_{\mathbf{s} / \mathbf{c}}\right)_{-1}+\left(\mathbf{8}_{\mathbf{s} / \mathbf{c}}\right)_{-1}+\left(\mathbf{8}_{\mathbf{s} / \mathbf{c}}\right)_{-3} .
\end{aligned}
$$

Hence, the BPS index 29 in table 1 should be interpreted as the representations $\mathbf{2 8}$ and $\mathbf{1}$, while 64 is interpreted as the representations $\mathbf{5} \mathbf{6}_{\mathbf{s} / \mathbf{c}}$ and $\mathbf{8}_{\mathbf{s} / \mathbf{c}}$.

Reversely, after assuming that the BPS indices are obtained by decomposing the so(10) representations to the subalgebra so( 8$) \times \mathrm{u}(1)$, with table 6 of the decomposition of various irreducible so(10) representations, we can check that no other candidate combinations of the so(10) representations can form the BPS indices 45 or 144 with the same decomposition. This is true also for the other BPS indices. We have listed the representations in table 1. Though in [37] the representations seem determined directly from the Weyl orbits, our determination of the representations is rather indirect through the decomposition.

It is known that the lattice points in the weight lattice with the identification of the root lattice are classified by the congruency class $\mathbb{Z}_{4}$ for so(10), so are the irreducible representations. It is interesting to further observe that the representations of so(10) appearing 


\begin{tabular}{|c|c|c|c|c|}
\hline$d$ & $\left(j_{\mathrm{L}}, j_{\mathrm{R}}\right)$ & BPS & $(-1)^{d-1} \sum_{|\boldsymbol{d}|=1}\left(N_{j_{\mathrm{L}}, j_{\mathrm{R}}}^{\boldsymbol{d}}\right)_{d^{+}-d^{-}}$ & representations \\
\hline 1 & $(0 ; 0)$ & 16 & $8_{+1}+8_{-1}$ & $\mathbf{1 6}$ \\
\hline 2 & $\left(0, \frac{1}{2}\right)$ & 10 & $1_{+2}+8_{0}+1_{-2}$ & $\mathbf{1 0}$ \\
\hline 3 & $(0,1)$ & 16 & $8_{+1}+8_{-1}$ & $\mathbf{1 6}$ \\
\hline \multirow{4}{*}{4} & $\left(0, \frac{1}{2}\right)$ & 1 & $1_{0}$ & $\mathbf{1}$ \\
\cline { 2 - 5 } & $\left(0, \frac{3}{2}\right)$ & 45 & $8_{+2}+29_{0}+8_{-2}$ & $\mathbf{4 5}$ \\
\cline { 2 - 5 } & $\left(\frac{1}{2}, 2\right)$ & 1 & $1_{0}$ & $\mathbf{1}$ \\
\hline \multirow{2}{*}{5} & $(0,1)$ & 16 & $8_{+1}+8_{-1}$ & $\mathbf{1 6}$ \\
\cline { 2 - 5 } & $(0,2)$ & 144 & $8_{+3}+64_{+1}+64_{-1}+8_{-3}$ & $\mathbf{1 4 4}$ \\
\cline { 2 - 5 } & $\left(\frac{1}{2}, \frac{5}{2}\right)$ & 16 & $8_{+1}+8_{-1}$ & $\mathbf{1 6}$ \\
\hline
\end{tabular}

Table 1. The BPS indices $N_{j_{\mathrm{L}}, j_{\mathrm{R}}}^{d}$ for $1 \leq d \leq 5$ of the $(2,2)$ model with the rank deformation $\mathrm{U}(N)_{k} \times \mathrm{U}(N+M)_{0} \times \mathrm{U}(N+2 M)_{-k} \times \mathrm{U}(N+M)_{0}$. The information on the non-vanishing BPS indices in the first three columns is recapitulated from the tables in [37] and the split into various degree differences in the fourth column comes from [36].

in the total degree $d$ are all the representations in the congruency class of $d \bmod 4$. For example, the representations appearing for odd $d$ are all fermionic ones with the dimensions being multiples of 16 . For this reason, from now on our tables of the decomposition of the so(10) representations and the characters in appendix A are listed by the congruency class.

This observation for the BPS indices of the $(2,2)$ model from the group-theoretical viewpoint may apply not just to the $(2,2)$ model. We also expect the group-theoretical viewpoint to work later in our study of the $(2,1)$ model.

\section{Topological string}

In this section we shall see that the instanton effects of the $(2,1)$ model are consistent with the free energy of topological strings (1.1). First we provide a set of four Kähler parameters which realizes the following structures of the instanton coefficients,

- the multi-covering structures of $d_{\ell}(2.8), \widetilde{b}_{2 \ell}(3.5)$ and $\widetilde{c}_{2 \ell-1}(2.12)$,

- the vanishing odd coefficients, $\widetilde{b}_{2 \ell-1}=0$, and

- the derivative relation between $\widetilde{c}_{2 \ell}$ and $\widetilde{b}_{2 \ell}(2.11)$.

Then we determine the BPS indices for small degrees. Interestingly, the BPS indices again correspond to the decomposition of the so(10) representations, where two differences of the degrees specifying the split of the BPS indices are identified with the two $u(1)$ charges in the decomposition to the subalgebra $\mathrm{so}(6) \times \mathrm{u}(1) \times \mathrm{u}(1)$. This is how the observations in section 3 are brought to life. Furthermore, once the representations are determined from the $(2,2)$ model, this enables us a top-down derivation for all of the instanton coefficients of the $(2,1)$ model. 


\subsection{Kähler parameters}

Our starting point is the same topological string free energy (1.1)

$$
\begin{aligned}
J^{\mathrm{WS}}\left(\mu_{\mathrm{eff}}\right) & =\sum_{j_{\mathrm{L}}, j_{\mathrm{R}}} \sum_{\boldsymbol{d}} N_{j_{\mathrm{L}}, j_{\mathrm{R}}}^{\boldsymbol{d}} \sum_{n=1}^{\infty} \frac{(-1)^{\left(s_{\mathrm{L}}+s_{\mathrm{R}}-1\right) n} s_{\mathrm{R}} \sin 2 \pi g_{\mathrm{s}} n s_{\mathrm{L}}}{n\left(2 \sin \pi g_{\mathrm{s}} n\right)^{2} \sin 2 \pi g_{\mathrm{s}} n} e^{-n \boldsymbol{d} \cdot \boldsymbol{T}}, \\
J^{\mathrm{MB}}\left(\mu_{\mathrm{eff}}\right) & =\sum_{j_{\mathrm{L}}, j_{\mathrm{R}}} \sum_{\boldsymbol{d}} N_{j_{\mathrm{L}}, j_{\mathrm{R}}}^{\boldsymbol{d}} \sum_{n=1}^{\infty} \frac{\partial}{\partial g_{\mathrm{s}}}\left[g_{\mathrm{s}} \frac{-\sin \frac{\pi n}{g_{\mathrm{s}}} s_{\mathrm{L}} \sin \frac{\pi n}{g_{\mathrm{s}}} s_{\mathrm{R}}}{4 \pi n^{2}\left(\sin \frac{\pi n}{g_{\mathrm{s}}}\right)^{3}} e^{-n \frac{\boldsymbol{d} \cdot \boldsymbol{T}}{g_{\mathrm{s}}}}\right] .
\end{aligned}
$$

The main assumption is to introduce the following four Kähler parameters

$$
T_{\uparrow}^{ \pm}=\frac{2 \mu_{\mathrm{eff}}}{k} \pm \pi i\left(1+\frac{1}{k}\right), \quad T_{\downarrow}^{ \pm}=\frac{2 \mu_{\mathrm{eff}}}{k} \pm \pi i\left(-1+\frac{1}{k}\right),
$$

with the string coupling constant identified as $g_{\mathrm{s}}=2 / k$. Due to the relation

$$
\boldsymbol{d} \cdot \boldsymbol{T}=d \frac{2 \mu_{\mathrm{eff}}}{k}+d_{\mathrm{m}} \pi i+d_{\mathrm{w}} \frac{\pi i}{k},
$$

with

$$
d=\sum_{ \pm}\left(d_{\uparrow}^{ \pm}+d_{\downarrow}^{ \pm}\right), \quad d_{\mathrm{m}}=\left(d_{\uparrow}^{+}-d_{\downarrow}^{+}\right)-\left(d_{\uparrow}^{-}-d_{\downarrow}^{-}\right), \quad d_{\mathrm{w}}=\left(d_{\uparrow}^{+}+d_{\downarrow}^{+}\right)-\left(d_{\uparrow}^{-}+d_{\downarrow}^{-}\right),
$$

we find that the whole information on the degrees $\boldsymbol{d}$ is simply encoded ${ }^{6}$ in the total degree $d$, the membrane degree $d_{\mathrm{m}}$ and the worldsheet degree $d_{\mathrm{w}}$. Hence, hereafter we sum the BPS indices over all degrees giving the same set of $\left(d, d_{\mathrm{w}}, d_{\mathrm{m}}\right)$ and label the BPS indices by these degrees

$$
N_{j_{\mathrm{L}}, j_{\mathrm{R}}}^{\left(d, d_{\mathrm{w}}, d_{\mathrm{m}}\right)}=\sum_{\left\{\boldsymbol{d} \mid\left(d, d_{\mathrm{w}}, d_{\mathrm{m}}\right)\right\}} N_{j_{\mathrm{L}}, j_{\mathrm{R}}}^{\boldsymbol{d}}
$$

For our later analysis we further assume the even property of $2 j_{\mathrm{L}}+2 j_{\mathrm{R}}-1-d$ and the symmetry of the BPS indices

$$
N_{j_{\mathrm{L}}, j_{\mathrm{R}}}^{\left(d, d_{\mathrm{w}}, d_{\mathrm{m}}\right)}=N_{j_{\mathrm{L}}, j_{\mathrm{R}}}^{\left(d,-d_{\mathrm{w}}, d_{\mathrm{m}}\right)} .
$$

Let us deduce the instanton coefficients from (4.1). As was noticed in [33], the imaginary part $\pm \pi i$ in the Kähler parameters (4.2) realizes the multi-covering structure of the worldsheet instanton $(2.8)$ when $2 j_{\mathrm{L}}+2 j_{\mathrm{R}}-1-d$ is even,

$$
J^{\mathrm{WS}}\left(\mu_{\mathrm{eff}}\right)=\sum_{m=1}^{\infty} d_{m} e^{-m \frac{2 \mu_{\mathrm{eff}}}{k}}, \quad d_{m}=\sum_{n \mid m} \frac{1}{n} \delta_{\frac{m}{n}}\left(\frac{k}{n}\right),
$$

where the multi-covering component of the worldsheet instanton is described by the BPS indices summed over all of the membrane degrees

$$
\delta_{d}(k)=\sum_{d_{\mathrm{w}}} \sum_{j_{\mathrm{L}}, j_{\mathrm{R}}} N_{j_{\mathrm{L}}, j_{\mathrm{R}}}^{\left(d, d_{\mathrm{\textrm {R }}}\right)}\left[\frac{s_{\mathrm{R}} \sin \frac{4 \pi}{k} s_{\mathrm{L}}}{\left(2 \sin \frac{2 \pi}{k}\right)^{2} \sin \frac{4 \pi}{k}} e^{-d_{\mathrm{w}} \frac{\pi i}{k}}\right], \quad N_{j_{\mathrm{L}}, j_{\mathrm{R}}}^{\left(d, d_{\mathrm{w}}\right)}=\sum_{d_{\mathrm{m}}} N_{j_{\mathrm{L}}, j_{\mathrm{R}}}^{\left(d, d_{\mathrm{w}}, d_{\mathrm{m}}\right)} .
$$

\footnotetext{
${ }^{6}$ The names of membrane degrees and worldsheet degrees will be clear in the later discussion. The even/odd parities of these degrees all coincide.
} 
The membrane instanton coefficients can be read off from (4.1) as

$$
J^{\mathrm{MB}}\left(\mu_{\mathrm{eff}}\right)=\sum_{\ell=1}^{\infty}\left(\widetilde{b}_{\ell} \mu_{\mathrm{eff}}+\widetilde{c}_{\ell}\right) e^{-\mu_{\mathrm{eff}}}
$$

with $\widetilde{b}_{\ell}$ and $\widetilde{c}_{\ell}$ given respectively by

$$
\widetilde{b}_{\ell}=\sum_{n d=\ell} \sum_{j_{\mathrm{L}}, j_{\mathrm{R}}} \sum_{d_{\mathrm{w}}} \sum_{d_{\mathrm{m}}} N_{j_{\mathrm{L}}, j_{\mathrm{R}}}^{\left(d, d_{\mathrm{m}}, d_{\mathrm{m}}\right)} e^{-n d_{\mathrm{m}} \frac{\pi k i}{2}} e^{-n d_{\mathrm{w}} \frac{\pi i}{2}} \frac{-d \sin \frac{\pi k n}{2} s_{\mathrm{L}} \sin \frac{\pi k n}{2} s_{\mathrm{R}}}{4 \pi n\left(\sin \frac{\pi k n}{2}\right)^{3}},
$$

and

$$
\begin{aligned}
\widetilde{c}_{\ell}=\sum_{n d=\ell} \sum_{j_{\mathrm{L}}, j_{\mathrm{R}}} \sum_{d_{\mathrm{w}}} \sum_{d_{\mathrm{m}}} N_{j_{\mathrm{L}}, j_{\mathrm{R}}}^{\left(d, d_{\mathrm{w}}, d_{\mathrm{m}}\right)} e^{-n d_{\mathrm{m}} \frac{\pi k i}{2}} e^{-n d_{\mathrm{w}} \frac{\pi i}{2}} \\
\quad \times\left(\frac{\pi i\left(k d_{\mathrm{m}}+d_{\mathrm{w}}\right)}{2}-k^{2} \frac{d}{d k} \frac{1}{k n}\right)\left[\frac{-\sin \frac{\pi k n}{2} s_{\mathrm{L}} \sin \frac{\pi k n}{2} s_{\mathrm{R}}}{4 \pi n\left(\sin \frac{\pi k n}{2}\right)^{3}}\right] .
\end{aligned}
$$

Now we can see the vanishing of odd coefficients $\widetilde{b}_{2 \ell-1}=0$ is realized from the symmetry of the BPS indices (4.6). This symmetry allows us to replace $e^{-n d_{\mathrm{w}} \frac{\pi i}{2}}$ in (4.10) with $\left(e^{-n d_{\mathrm{w}} \frac{\pi i}{2}}+e^{n d_{\mathrm{w}} \frac{\pi i}{2}}\right) / 2$, which vanishes when $n d=2 \ell-1$ is odd since $n, d, d_{\mathrm{w}}$ are all odd. Moreover, for $\widetilde{b}_{2 \ell}$, by noticing

$$
\left.\frac{e^{-n d_{\mathrm{w}} \frac{\pi i}{2}}+e^{n d_{\mathrm{w}} \frac{\pi i}{2}}}{2}\right|_{n d=2 \ell}= \begin{cases}(-1)^{\ell}, & \text { for even } n, \\ (-1)^{\frac{d_{\mathrm{w}}}{2}}, & \text { for odd } n,\end{cases}
$$

we obtain the following multi-covering structure

$$
\tilde{b}_{2 \ell}=\sum_{n \mid 2 \ell, n \in 2 \mathbb{N}} \frac{(-1)^{\ell}}{n} \beta_{\frac{2 \ell}{n}}(n k)+\sum_{n \mid 2 \ell, n \in 2 \mathbb{N}-1} \frac{1}{n} \beta_{\frac{2 \ell}{n}}^{\prime}(n k),
$$

which is exactly what we have suggested in (3.5). Here the multi-covering components are

$$
\begin{aligned}
& \beta_{d}(k)=\sum_{j_{\mathrm{L}}, j_{\mathrm{R}}} \sum_{d_{\mathrm{m}}} N_{j_{\mathrm{L}}, j_{\mathrm{R}}}^{\left(d, d_{\mathrm{m}}\right)} \frac{-d \sin \frac{\pi k}{2} s_{\mathrm{L}} \sin \frac{\pi k}{2} s_{\mathrm{R}}}{4 \pi\left(\sin \frac{\pi k}{2}\right)^{3}} e^{-d_{\mathrm{m}} \frac{\pi k i}{2}} \\
& \beta_{d}^{\prime}(k)=\sum_{j_{\mathrm{L}}, j_{\mathrm{R}}} \sum_{d_{\mathrm{m}}} N_{j_{\mathrm{L}}, j_{\mathrm{R}}}^{\prime\left(d, d_{\mathrm{m}}\right)} \frac{-d \sin \frac{\pi k}{2} s_{\mathrm{L}} \sin \frac{\pi k}{2} s_{\mathrm{R}}}{4 \pi\left(\sin \frac{\pi k}{2}\right)^{3}} e^{-d_{\mathrm{m}} \frac{\pi k i}{2}},
\end{aligned}
$$

with the original BPS indices $N_{j_{\mathrm{L}}, j_{\mathrm{R}}}^{\left(d, d_{\mathrm{m}}\right)}$ and the alternating BPS indices $N_{j_{\mathrm{L}}, j_{\mathrm{R}}}^{\left(d, d_{\mathrm{m}}\right)}$ defined as

$$
N_{j_{\mathrm{L}}, j_{\mathrm{R}}}^{\left(d, d_{\mathrm{m}}\right)}=\sum_{d_{\mathrm{w}}} N_{j_{\mathrm{L}}, j_{\mathrm{R}}}^{\left(d, d_{\mathrm{w}}, d_{\mathrm{m}}\right)}, \quad N_{j_{\mathrm{L}}, j_{\mathrm{R}}}^{\left(d, d_{\mathrm{m}}\right)}=\left(\sum_{d_{\mathrm{w}} \equiv 0(\bmod 4)}-\sum_{d_{\mathrm{w}} \equiv 2(\bmod 4)}\right) N_{j_{\mathrm{L}}, j_{\mathrm{R}}}^{\left(d, d_{\mathrm{w}}, d_{\mathrm{m}}\right)}
$$

The coefficient $\widetilde{c}_{\ell}(4.11)$ can be simplified in the same way. For even instantons $\widetilde{c}_{2 \ell}$, from the symmetry of the BPS indices (4.6), the $\pi i d_{\mathrm{w}} / 2$ term is cancelled and the $\pi i k d_{\mathrm{m}} / 2$ term is combined into the derivative term to reproduce the derivative relation (2.11). For 
odd instantons $\widetilde{c}_{2 \ell-1}$, on the other hand, from the symmetry of the BPS indices (4.6), only the $\pi i d_{\mathrm{w}} / 2$ term survives. Due to the simplification

$$
\left.\frac{i d_{\mathrm{w}} e^{-n d_{\mathrm{w}} \frac{\pi i}{2}}-i d_{\mathrm{w}} e^{n d_{\mathrm{w}} \frac{\pi i}{2}}}{2}\right|_{n d=2 \ell-1}=(-1)^{\frac{n-1}{2}+\frac{d_{\mathrm{w}}-1}{2}} d_{\mathrm{w}},
$$

(which can be proved from $\left(e^{ \pm \frac{\pi i}{2}}\right)^{(n-1)\left(d_{\mathrm{w}}-1\right)}=1$ by noting both $n$ and $d_{\mathrm{w}}$ are odd if $n d$ is odd), we reproduce the multi-covering structure (2.12)

$$
\widetilde{c}_{2 \ell-1}=\sum_{n \mid 2 \ell-1} \frac{(-1)^{\frac{n-1}{2}}}{n} \gamma_{\frac{2 \ell-1}{n}}(n k),
$$

where the multi-covering components are

$$
\gamma_{d}(k)=\sum_{j_{\mathrm{L}}, j_{\mathrm{R}}} \sum_{d_{\mathrm{m}}} M_{j_{\mathrm{L}}, j_{\mathrm{R}}}^{\left(d, d_{\mathrm{m}}\right)} \frac{-\sin \frac{\pi k}{2} s_{\mathrm{L}} \sin \frac{\pi k}{2} s_{\mathrm{R}}}{8\left(\sin \frac{\pi k}{2}\right)^{3}} e^{-d_{\mathrm{m}} \frac{\pi k i}{2}},
$$

with the weighted BPS indices $M_{j_{\mathrm{L}}, j_{\mathrm{R}}}^{\left(d, d_{\mathrm{m}}\right)}$

$$
M_{j_{\mathrm{L}}, j_{\mathrm{R}}}^{\left(d, d_{\mathrm{m}}\right)}=\sum_{d_{\mathrm{w}}}(-1)^{\frac{d_{\mathrm{w}}-1}{2}} d_{\mathrm{w}} N_{j_{\mathrm{L}}, j_{\mathrm{R}}}^{\left(d, d_{\mathrm{w}}, d_{\mathrm{m}}\right)}
$$

\subsection{BPS indices}

After constructing the general framework to reproduce the multi-covering structure and the derivative relation, now we can ask whether the expression of the topological string free energy matches with the instanton coefficients if we choose the BPS indices suitably. As in [36] we shall assume the positivity $(-1)^{d-1} N_{j_{\mathrm{L}}, j_{\mathrm{R}}}^{\left(d, d_{\mathrm{m}}, d_{\mathrm{m}}\right)} \geq 0$ and study how the original total BPS indices listed in [37] is partitioned

$$
N_{j_{\mathrm{L}}, j_{\mathrm{R}}}^{d}=\sum_{d_{\mathrm{w}}} \sum_{d_{\mathrm{m}}} N_{j_{\mathrm{L}}, j_{\mathrm{R}}}^{\left(d, d_{\mathrm{w}}, d_{\mathrm{m}}\right)}
$$

We have observed in (3.2) that the worldsheet instantons of the rank deformed $(2,2)$ model agree with those of the $(2,1)$ model if we rescale $k$ by $1 / 2$ and set $M= \pm 1 / 2$. Hence, if the worldsheet BPS indices $N_{j_{\mathrm{L}}, j_{\mathrm{R}}}^{\left(d, d_{\mathrm{w}}\right)}$ are those identified in table 1 , this expression automatically reproduces the worldsheet instantons of the $(2,1)$ model. Also, since we have brought the expression of $\beta_{d}(k)$ from the $(2,2)$ model as in $(3.7)$, we also hope to identify the membrane BPS indices $N_{j_{\mathrm{L}}, j_{\mathrm{R}}}^{\left(d, d_{\mathrm{m}}\right)}$ to be those in table 1.

For $d=1,2,3$, since there is only one type of spins for each degree we find the identification

$$
M_{0,0}^{(1, \pm 1)}=4, \quad N_{0, \frac{1}{2}}^{\prime(2, \pm 2)}=-1, \quad N_{0, \frac{1}{2}}^{\prime(2,0)}=-4, \quad M_{0,1}^{(3, \pm 1)}=4
$$

from the comparison of the general expression (4.14), (4.18) with $\gamma_{1}(k), \beta_{2}^{\prime}(k), \gamma_{3}(k)$. Combining with the condition of the total worldsheet BPS indices $N_{j_{\mathrm{L}}, j_{\mathrm{R}}}^{\left(d, d_{\mathrm{w}}\right)}$ and the total 
membrane BPS indices $N_{j_{\mathrm{L}}, j_{\mathrm{R}}}^{\left(d, d_{\mathrm{m}}\right)}$, both of which are given by

$$
\begin{aligned}
& N_{0,0}^{\left(1, d_{\mathrm{w}}= \pm 1\right)}=8, \quad N_{0, \frac{1}{2}}^{\left(2, d_{\mathrm{w}}= \pm 2\right)}=-1, \quad N_{0, \frac{1}{2}}^{\left(2, d_{\mathrm{w}}=0\right)}=-8, \quad N_{0,1}^{\left(3, d_{\mathrm{w}}= \pm 1\right)}=8, \\
& N_{0,0}^{\left(1, d_{\mathrm{m}}= \pm 1\right)}=8, \quad N_{0, \frac{1}{2}}^{\left(2, d_{\mathrm{m}}= \pm 2\right)}=-1, \quad N_{0, \frac{1}{2}}^{\left(2, d_{\mathrm{m}}=0\right)}=-8, \quad N_{0,1}^{\left(3, d_{\mathrm{m}}= \pm 1\right)}=8,
\end{aligned}
$$

we find that the separated BPS indices $N_{j_{\mathrm{L}}, j_{\mathrm{R}}}^{\left(d, d_{\mathrm{m}}, d_{\mathrm{m}}\right)}$ are

$$
N_{0,0}^{(1, \pm 1, \pm 1)}=4, \quad N_{0, \frac{1}{2}}^{(2, \pm 2,0)}=N_{0, \frac{1}{2}}^{(2,0, \pm 2)}=-1, \quad N_{0, \frac{1}{2}}^{(2,0,0)}=-6, \quad N_{0,1}^{(3, \pm 1, \pm 1)}=4 .
$$

Looking closely at the decomposition for $d=2$, for example, we find that the membrane BPS index $\left|N_{0, \frac{1}{2}}^{\left(2, d_{\mathrm{m}}=0\right)}\right|=8$ is split into

$$
8 \rightarrow 1_{+2}+6_{0}+1_{-2}
$$

where we have denoted the worldsheet degree $d_{\mathrm{w}}$ of $N_{j_{\mathrm{L}}, j_{\mathrm{R}}}^{\left(d, d_{\mathrm{w}}, d_{\mathrm{m}}\right)}$ as subscripts. Then, this expression is reminiscent of the decomposition of the representation $\mathbf{8}_{\mathbf{v}}$ from so(8) to the subalgebra so(6) $\times \mathrm{u}(1)$. This interpretation works for the other BPS indices in $d=1,2,3$ as well.

After observing the relation to the further decomposition of the so(8) representations to $\mathrm{so}(6) \times \mathrm{u}(1)$, since we have already identified the BPS indices as the representations of so(10) for $d=4,5$ in table 1 , the only remaining task is to decompose each so(8) representation in $(3.10)$ to $\mathrm{so}(6) \times \mathrm{u}(1)$,

$$
\begin{aligned}
\mathbf{2 8} & \rightarrow(\mathbf{6})_{+2}+(\mathbf{1 5})_{0}+(\mathbf{1})_{0}+(\mathbf{6})_{-2}, \quad \mathbf{8}_{\mathbf{v}} \rightarrow(\mathbf{1})_{+2}+(\mathbf{6})_{0}+(\mathbf{1})_{-2}, \quad \mathbf{1} \rightarrow(\mathbf{1})_{0}, \quad(4 . \\
\mathbf{5 6}_{\mathbf{s} / \mathbf{c}} & \rightarrow(\mathbf{4})_{+3}+(\overline{\mathbf{2 0}})_{+1}+(\overline{\mathbf{4}})_{+1}+(\mathbf{2 0})_{-1}+(\mathbf{4})_{-1}+(\overline{\mathbf{4}})_{-3}, \quad \mathbf{\mathbf { 8 } _ { \mathbf { s } / \mathbf { c } }} \rightarrow(\overline{\mathbf{4}})_{+1}+(\mathbf{4})_{-1} .
\end{aligned}
$$

Then we find that the degrees should be decomposed as

$$
\begin{array}{rlrl}
N_{0, \frac{3}{2}}^{(4,0,0)} & =-17, & N_{0, \frac{3}{2}}^{(4,0, \pm 2)} & =N_{0, \frac{3}{2}}^{(4, \pm 2,0)}=-6, \quad N_{0, \frac{3}{2}}^{(4, \pm 2, \pm 2)}=-1, \\
N_{0,2}^{(5, \pm 3, \pm 1)} & =N_{0,2}^{(5, \pm 1, \pm 3)}=28, \quad N_{0,2}^{(5, \pm 3, \pm 3)}=4,
\end{array}
$$

which gives the alternating BPS indices and the weighted BPS indices

$$
\begin{array}{rlrl}
N_{0, \frac{3}{2}}^{\prime(4,0)} & =-5, & N_{0, \frac{3}{2}}^{\prime(42)}=-4, \\
M_{0,2}^{(5, \pm 1)}=32, & M_{0,2}^{(5, \pm 3)}=8 .
\end{array}
$$

Substituting these BPS indices into (4.14) and (4.18), we find that the instanton coefficients in (A.3) and (A.2) obtained from the WKB expansions are beautifully reproduced.

To summarize, our proposal is that the reduced grand potential of the $(2,1)$ model is described by the BPS indices which are obtained by identifying the total BPS indices of the local $D_{5}$ del Pezzo geometry as the representations of so(10) and decomposing the so(10) representations to the subalgebra $\mathrm{so}(6) \times \mathrm{u}(1) \times \mathrm{u}(1)$ with the two $\mathrm{u}(1)$ charges identified as the two degree differences. 


\subsection{Characters}

We have found that we can describe the reduced grand potential of the $(2,1)$ model by the free energy of topological strings if we adopt the ansatz of the four Kähler parameters (4.2) and choose the BPS indices appropriately by the decomposition of the so(10) representations. Here we point out that our proposal on the reduced grand potential can be summarized compactly in terms of the characters of so(10).

For this purpose, we first introduce the characters of so(10) with two fugacities,

$$
\chi_{\mathbf{R}}(p, q)=\sum_{d_{\mathrm{w}}, d_{\mathrm{m}}} p^{d_{\mathrm{w}}} q^{d_{\mathrm{m}}} \operatorname{dim}\left(\mathbf{r}_{\left(d_{\mathrm{w}}, d_{\mathrm{m}}\right)}\right),
$$

each of which measures the two $\mathrm{u}(1)$ charges in the decomposition

$$
\mathrm{so}(10) \rightarrow \operatorname{so}(6) \times \mathrm{u}(1) \times \mathrm{u}(1), \quad \mathbf{R} \rightarrow \sum \mathbf{r}_{\left(d_{\mathrm{w}}, d_{\mathrm{m}}\right)} .
$$

Then, once the total BPS index is identified as the so(10) representations,

$$
(-1)^{d-1} N_{j_{\mathrm{L}}, j_{\mathrm{R}}}^{d}=\sum_{\mathbf{R}} n_{j_{\mathrm{L}}, j_{\mathrm{R}}}^{d, \mathbf{R}} \operatorname{dim}(\mathbf{R})
$$

each BPS index coming from the so(10) representations can be given as

$$
\begin{aligned}
(-1)^{d-1} \sum_{d_{\mathrm{w}}} N_{j_{\mathrm{L}}, j_{\mathrm{R}}}^{\left(d, d_{\mathrm{w}}\right)} p^{d_{\mathrm{w}}}=\sum_{\mathbf{R}} n_{j_{\mathrm{L}}, j_{\mathrm{R}}}^{d, \mathbf{R}} \chi_{\mathbf{R}}(p, 1), \quad(-1)^{d-1} \sum_{d_{\mathrm{m}}} N_{j_{\mathrm{L}}, j_{\mathrm{R}}}^{\left(d, d_{\mathrm{m}}\right)} q^{d_{\mathrm{m}}}=\sum_{\mathbf{R}} n_{j_{\mathrm{L}}, j_{\mathrm{R}}}^{d, \mathbf{R}} \chi_{\mathbf{R}}(1, q), \\
(-1)^{d-1} \sum_{d_{\mathrm{m}}} N_{j_{\mathrm{L}}, j_{\mathrm{R}}}^{\prime\left(d, d_{\mathrm{m}}\right)} q^{d_{\mathrm{m}}}=\sum_{\mathbf{R}} n_{j_{\mathrm{L}}, j_{\mathrm{R}}}^{d, \mathbf{R}} \chi_{\mathbf{R}}(i, q), \quad(-1)^{d-1} \sum_{d_{\mathrm{m}}} M_{j_{\mathrm{L}}, j_{\mathrm{R}}}^{\left(d, d_{\mathrm{m}}\right)} q^{d_{\mathrm{m}}}=\sum_{\mathbf{R}} n_{j_{\mathrm{L}}, j_{\mathrm{R}}}^{d, \mathbf{R}} \frac{\partial \chi_{\mathbf{R}}}{\partial p}(i, q) .
\end{aligned}
$$

This implies from (4.8), (4.14), (4.18) that the multi-covering components of the worldsheet instantons and the membrane instantons are compactly given in terms of the characters by

$$
\begin{aligned}
& \delta_{d}(k)=\frac{(-1)^{d-1}}{\left(2 \sin \frac{2 \pi}{k}\right)^{2}} \sum_{j_{\mathrm{L}}, j_{\mathrm{R}}} \sum_{\mathbf{R}} n_{j_{\mathrm{L}}, j_{\mathrm{R}}}^{d, \mathbf{R}} \chi_{\mathbf{R}}\left(e^{-\frac{\pi i}{k}}, 1\right) \chi_{j_{\mathrm{L}}}\left(e^{\frac{4 \pi i}{k}}\right) \chi_{j_{\mathrm{R}}}(1), \\
& \beta_{d}(k)=\frac{(-1)^{d} d}{4 \pi \sin \frac{\pi k}{2}} \sum_{j_{\mathrm{L}}, j_{\mathrm{R}}} \sum_{\mathbf{R}} n_{j_{\mathrm{L}}, j_{\mathrm{R}}}^{d, \mathbf{R}} \chi_{\mathbf{R}}\left(1, e^{-\frac{\pi k i}{2}}\right) \chi_{j_{\mathrm{L}}}\left(e^{\frac{\pi k i}{2}}\right) \chi_{j_{\mathrm{R}}}\left(e^{\frac{\pi k i}{2}}\right), \\
& \beta_{d}^{\prime}(k)=\frac{(-1)^{d} d}{4 \pi \sin \frac{\pi k}{2}} \sum_{j_{\mathrm{L}}, j_{\mathrm{R}}} \sum_{\mathbf{R}} n_{j_{\mathrm{L}}, j_{\mathrm{R}}}^{d, \mathbf{R}} \chi_{\mathbf{R}}\left(i, e^{-\frac{\pi k i}{2}}\right) \chi_{j_{\mathrm{L}}}\left(e^{\frac{\pi k i}{2}}\right) \chi_{j_{\mathrm{R}}}\left(e^{\frac{\pi k i}{2}}\right), \\
& \gamma_{d}(k)=\frac{(-1)^{d}}{8 \sin \frac{\pi k}{2}} \sum_{j_{\mathrm{L}}, j_{\mathrm{R}}} \sum_{\mathbf{R}} n_{j_{\mathrm{L}}, j_{\mathrm{R}}}^{d, \mathbf{R}} \frac{\partial \chi_{\mathbf{R}}}{\partial p}\left(i, e^{-\frac{\pi k i}{2}}\right) \chi_{j_{\mathrm{L}}}\left(e^{\frac{\pi k i}{2}}\right) \chi_{j_{\mathrm{R}}}\left(e^{\frac{\pi k i}{2}}\right),
\end{aligned}
$$

where we have also introduced the $\mathrm{su}(2)$ character

$$
\chi_{j}(q)=\frac{q^{2 j+1}-q^{-(2 j+1)}}{q-q^{-1}} .
$$




\begin{tabular}{|c|c|c|c|}
\hline$d$ & $\left(j_{\mathrm{L}}, j_{\mathrm{R}}\right)$ & BPS & representations \\
\hline 6 & $\left(0, \frac{1}{2}\right)$ & 10 & $\mathbf{1 0}$ \\
\cline { 2 - 4 } & $\left(0, \frac{3}{2}\right)$ & 130 & $\mathbf{1 2 0}+\mathbf{1 0}$ \\
\cline { 2 - 4 } & $\left(0, \frac{5}{2}\right)$ & 456 & $\mathbf{3 2 0}+\mathbf{1 2 6}+\mathbf{1 0}$ \\
\cline { 2 - 4 } & $\left(\frac{1}{2}, 2\right)$ & 10 & $\mathbf{1 0}$ \\
\cline { 2 - 4 } & $\left(\frac{1}{2}, 3\right)$ & 130 & $\mathbf{1 2 0}+\mathbf{1 0}$ \\
\cline { 2 - 4 } & $\left(1, \frac{7}{2}\right)$ & 10 & $\mathbf{1 0}$ \\
\hline
\end{tabular}

\begin{tabular}{|c|c|c|c|}
\hline$d$ & $\left(j_{\mathrm{L}}, j_{\mathrm{R}}\right)$ & BPS & representations \\
\hline \multirow[t]{11}{*}{7} & $(0,0)$ & 16 & 16 \\
\hline & $(0,1)$ & 160 & $144+16$ \\
\hline & $(0,2)$ & 736 & $560+144+2 \times 16$ \\
\hline & $(0,3)$ & 1440 & $720+560+144+16$ \\
\hline & $(0,4)$ & 16 & 16 \\
\hline & $\left(\frac{1}{2}, \frac{3}{2}\right)$ & 16 & 16 \\
\hline & $\left(\frac{1}{2}, \frac{5}{2}\right)$ & 176 & $144+2 \times 16$ \\
\hline & $\left(\frac{1}{2}, \frac{7}{2}\right)$ & 736 & $560+144+2 \times 16$ \\
\hline & $(1,3)$ & 16 & 16 \\
\hline & $(1,4)$ & 160 & $144+16$ \\
\hline & $\left(\frac{3}{2}, \frac{9}{2}\right)$ & 16 & 16 \\
\hline
\end{tabular}

Table 2. The constituent representations for the total BPS indices of the $(2,2)$ model for $d=6,7$.

\subsection{Higher degrees}

We believe that all the evidences we have provided in section 4.2 are already quite nontrivial. Nevertheless, in this subsection we shall proceed to even higher degrees $d=6,7,8$ to convince the readers completely of our proposal.

After proposing to obtain the BPS indices from the decomposition of the representations, our remaining task is to identify the so(10) representations which the total BPS indices listed in [37] consist of and to decompose the representations to the subalgebra so $(8) \times u(1)$. This can be done completely in the study of the $(2,2)$ model before considering the $(2,1)$ model. Then, we can apply our rule of further decomposing the so(8) representations to the subalgebra $\mathrm{so}(6) \times \mathrm{u}(1)$ to see whether the predicted worldsheet instantons coincide with those of the $(2,1)$ model obtained from the numerical fitting in (A.1) and whether the predicted membrane instantons coincide with those of the $(2,1)$ model obtained from the WKB expansion in (A.2) and (A.3). Hence we start our analysis purely on the $(2,2)$ model.

For $d=6$ we can study either from the numerical values of the worldsheet instantons of the $(2,2)$ model or the WKB expansion for the membrane instantons. In either method, we assume that the total BPS index 456 in the spin $\left(0, \frac{5}{2}\right)$ can be given by an integral linear combination of all the representations in the congruency class of $6 \equiv 2 \bmod 4$ with the dimensions smaller than or equal to 456 (which are 10, 120, 126, 210' ${ }^{\prime}$ and $\mathbf{3 2 0}$ ), while the total BPS indices 130 in the spins $\left(0, \frac{3}{2}\right)$ and $\left(\frac{1}{2}, 3\right)$ are given by other linear combinations of 10, 120 and 126. Then, for the former method, we ask which combination gives correctly the numerical values listed in appendix C.1.3 of [36], while for the latter method, we ask which combination gives correctly the WKB expansion in (A.5). In either method, we obtain the result in table 2 . For $d=7$ we need to utilize both the numerical values of the 


\begin{tabular}{|c|c|c|c|}
\hline$d$ & $\left(j_{\mathrm{L}}, j_{\mathrm{R}}\right)$ & BPS & representations \\
\hline \multirow[t]{10}{*}{8} & $\left(0, \frac{7}{2}\right)$ & 4726 & $\mathbf{1 3 8 6}+\mathbf{1 0 5 0}+2 \times \mathbf{9 4 5}+\mathbf{2 1 0}+\mathbf{5 4}+3 \times \mathbf{4 5}+\mathbf{1}$ \\
\hline & $\left(0, \frac{5}{2}\right),\left(\frac{1}{2}, 4\right)$ & 3431 & $\mathbf{1 0 5 0}+\mathbf{9 4 5}+\mathbf{7 7 0}+2 \times \mathbf{2 1 0}+2 \times \mathbf{5 4}+3 \times \mathbf{4 5}+3 \times \mathbf{1}$ \\
\hline & $\left(\frac{1}{2}, 3\right)$ & 1602 & $\mathbf{9 4 5}+2 \times \mathbf{2 1 0}+\mathbf{5 4}+4 \times \mathbf{4 5}+3 \times \mathbf{1}$ \\
\hline & $\left(0, \frac{3}{2}\right),\left(1, \frac{9}{2}\right)$ & 1345 & $\mathbf{9 4 5}+\mathbf{2 1 0}+\mathbf{5 4}+3 \times \mathbf{4 5}+\mathbf{1}$ \\
\hline & $\left(\frac{1}{2}, 2\right),\left(1, \frac{7}{2}\right)$ & 357 & $\mathbf{2 1 0}+\mathbf{5 4}+2 \times \mathbf{4 5}+3 \times \mathbf{1}$ \\
\hline & $\left(0, \frac{1}{2}\right),\left(\frac{3}{2}, 5\right)$ & 311 & $210+54+45+2 \times 1$ \\
\hline & $\left(0, \frac{9}{2}\right)$ & 257 & $210+45+2 \times 1$ \\
\hline & $\begin{array}{l}\left(\frac{1}{2}, 1\right),\left(\frac{1}{2}, 5\right), \\
\left(1, \frac{5}{2}\right),\left(\frac{3}{2}, 4\right)\end{array}$ & 46 & $45+1$ \\
\hline & $\left(2, \frac{11}{2}\right)$ & 45 & 45 \\
\hline & $\begin{array}{c}\left(1, \frac{3}{2}\right),\left(1, \frac{11}{2}\right),\left(\frac{3}{2}, 3\right), \\
\left(2, \frac{9}{2}\right),\left(\frac{5}{2}, 6\right)\end{array}$ & 1 & 1 \\
\hline
\end{tabular}

Table 3. The constituent representations for the total BPS indices of the $(2,2)$ model for $d=8$.

worldsheet instantons in appendix C.1.3 of [36] and the WKB expansion of the membrane instantons in (A.5). With both the data we can again fix exactly which representations appear in the total BPS indices. The results are listed in table 2.

For $d=6,7$ we can substitute the BPS indices into the worldsheet instanton to find

$$
\begin{aligned}
\delta_{6}(k, M)= & -\frac{756+579 \cos \frac{2 M \pi}{k}+24 \cos \frac{4 M \pi}{k}}{\sin ^{2} \frac{\pi}{k}}+\left(800+480 \cos \frac{2 M \pi}{k}\right) \\
& -\left(256+64 \cos \frac{2 M \pi}{k}\right) \sin ^{2} \frac{\pi}{k}, \\
\delta_{7}(k, M)= & \frac{7112 \cos \frac{M \pi}{k}+1288 \cos \frac{3 M \pi}{k}+28 \cos \frac{5 M \pi}{k}}{\sin ^{2} \frac{\pi}{k}}-\left(13120 \cos \frac{M \pi}{k}+1696 \cos \frac{3 M \pi}{k}\right) \\
& +\left(9472 \cos \frac{M \pi}{k}+576 \cos \frac{3 M \pi}{k}\right) \sin ^{2} \frac{\pi}{k}-2560 \cos \frac{M \pi}{k} \sin ^{4} \frac{\pi}{k} .
\end{aligned}
$$

We find that we can obtain the worldsheet instanton of the $(2,1)$ model (A.1) by substituting $M= \pm 1 / 2$ and replacing $k$ by $k / 2$ as in (3.2). By applying this rule we encounter the cosine functions with higher arguments which can be reexpanded by the denominator, as we have explained below (3.2). Due to this reason, the rule observed in [26] should be modified by (3.2).

Now with the characters in appendix A.3 which computes the alternating BPS indices $N_{j_{\mathrm{L}}, j_{\mathrm{R}}}^{\prime\left(d, d_{\mathrm{m}}\right)}$ and the weighted BPS indices $M_{j_{\mathrm{L}}, j_{\mathrm{R}}}^{\left(d, d_{\mathrm{m}}\right)}$ for various so(10) representations, we can predict the membrane instantons of the $(2,1)$ model for $d=6,7$. We find a very non-trivial match with those of the $(2,1)$ model (A.2) and (A.3) obtained by the WKB expansion.

For $d=8$, since there are more degrees of freedom to identify the representations, we need to impose one more assumption. In the table of [37] the total BPS indices 3431 appear in both the spins $\left(0, \frac{5}{2}\right)$ and $\left(\frac{1}{2}, 4\right)$. We assume that the same numbers of the BPS indices in different spins are identified as the same combination of the so(10) representations. Under 
this assumption, we find only two solutions. Aside from the one listed in table 3, the other solution is to replace the representations for the total BPS indices 3431 by

$$
2 \times \mathbf{1 0 5 0}+\mathbf{9 4 5}+2 \times \mathbf{5 4}+3 \times \mathbf{4 5}+143 \times \mathbf{1} .
$$

From the characters in (A.11), we find that only the set of representations listed in table 3 correctly reproduces the membrane instanton coefficient of the $(2,1)$ model (A.3) obtained by the WKB expansion.

In the above identification of the representations for the BPS indices of $d=8$, we have adopted the assumption that the same BPS indices consist of the same set of the so(10) representations. Since we do not have a persuasive reason for this assumption, we have also performed an alternative analysis. Namely, instead of the above assumption, we adopt our proposal of the relation between the $(2,2)$ model and the decomposition of the so(10) representations to $\mathrm{so}(8) \times \mathrm{u}(1)$ and the relation between the $(2,1)$ model and the decomposition of the same representations to so $(6) \times u(1) \times u(1)$ simultaneously. Then, we reach the same result of the identification of the so(10) representation listed in table 3.

\section{Rank-deformed $(2,2)$ model from characters}

Previously in [36] two types of rank deformations in the $(2,2)$ model were studied. As we have seen in section 3.3, one of the rank deformations $\mathrm{U}(N)_{k} \times \mathrm{U}(N+M)_{0} \times \mathrm{U}(N+2 M)_{-k} \times \mathrm{U}(N+M)_{0}$ corresponds to introducing the fugacity to distinguish the $\mathrm{u}(1)$ charge in the decomposition of the $\mathrm{so}(10)$ representations to the subalgebra $\mathrm{so}(8) \times \mathrm{u}(1)$. Here let us turn to revisiting the two-parameter rank deformation $\mathrm{U}\left(N+M_{\mathrm{II}}\right)_{k} \times \mathrm{U}\left(N+M_{\mathrm{I}}\right)_{0} \times \mathrm{U}\left(N+2 M_{\mathrm{I}}+M_{\mathrm{II}}\right)_{-k} \times \mathrm{U}\left(N+M_{\mathrm{I}}\right)_{0}$ in [36] where the previous deformation corresponds to $\left(M_{\mathrm{I}}, M_{\mathrm{II}}\right)=(M, 0)$.

To describe this deformation, in [36] six Kähler parameters were identified

$$
\begin{aligned}
& T_{1}^{ \pm}=\frac{\mu_{\mathrm{eff}}}{k} \pm \pi i\left(1-\frac{M_{\mathrm{I}}}{k}-\frac{2 M_{\mathrm{II}}}{k}\right), \\
& T_{2}^{ \pm}=\frac{\mu_{\mathrm{eff}}}{k} \pm \pi i\left(1-\frac{M_{\mathrm{I}}}{k}\right), \\
& T_{3}^{ \pm}=\frac{\mu_{\mathrm{eff}}}{k} \pm \pi i\left(1-\frac{M_{\mathrm{I}}}{k}+\frac{2 M_{\mathrm{II}}}{k}\right),
\end{aligned}
$$

and the corresponding BPS indices were studied. It was difficult to distribute the BPS indices into various degrees precisely, which is essentially due to the relations among the Kähler parameters

$$
2 T_{2}^{ \pm}=T_{1}^{ \pm}+T_{3}^{ \pm}, \quad T_{1}^{+}+T_{1}^{-}=T_{2}^{+}+T_{2}^{-}=T_{3}^{+}+T_{3}^{-}, \quad T_{2}^{ \pm}+T_{1}^{\mp}=T_{2}^{\mp}+T_{3}^{ \pm} .
$$

In other words, the description in [36] with the six Kähler parameters is probably correct though it may not be the most economical description because the six Kähler parameters are too abundant for the deformation with only two parameters. Our studies in the previous section suggest that instead of introducing many Kähler parameters it is more economical 


\begin{tabular}{|c|c|c|c|c|}
\hline$d$ & $\left(j_{\mathrm{L}}, j_{\mathrm{R}}\right)$ & $d_{\mathrm{I}}$ & $\mathrm{BPS}$ & $(-1)^{d-1} \sum_{d_{\mathrm{II}}}\left(N_{j_{\mathrm{L}}, j_{\mathrm{R}}}^{\left(d, d_{\mathrm{I}}, d_{\mathrm{II}}\right)}\right)_{d_{\mathrm{II}}}$ \\
\hline 1 & $(0 ; 0)$ & \pm 1 & 8 & $2_{+1}+4_{0}+2_{-1}$ \\
\hline \multirow{2}{*}{2} & \multirow{2}{*}{$\left(0, \frac{1}{2}\right)$} & 0 & 8 & $2_{+1}+4_{0}+2_{-1}$ \\
\cline { 3 - 5 } & & \pm 2 & 1 & $1_{0}$ \\
\hline \multirow{2}{*}{3} & $(0,1)$ & \pm 1 & 8 & $2_{+1}+4_{0}+2_{-1}$ \\
\hline \multirow{4}{*}{4} & $\left(0, \frac{1}{2}\right)$ & 0 & 1 & $1_{0}$ \\
\cline { 2 - 5 } & $\left(0, \frac{3}{2}\right)$ & 0 & 29 & $1_{+2}+8_{+1}+11_{0}+8_{-1}+1_{-2}$ \\
\cline { 3 - 5 } & & \pm 2 & 8 & $2_{+1}+4_{0}+2_{-1}$ \\
\cline { 2 - 5 } & $\left(\frac{1}{2}, 2\right)$ & 0 & 1 & $1_{0}$ \\
\hline
\end{tabular}

Table 4. The BPS indices $N_{j_{\mathrm{L}}, j_{\mathrm{R}}}^{d}$ for $1 \leq d \leq 4$ of the $(2,2)$ model with the rank deformation $\mathrm{U}\left(N+M_{\mathrm{II}}\right)_{k} \times \mathrm{U}\left(N+M_{\mathrm{I}}\right)_{0} \times \mathrm{U}\left(N+2 M_{\mathrm{I}}+M_{\mathrm{II}}\right)_{-k} \times \mathrm{U}\left(N+M_{\mathrm{I}}\right)_{0}$. The table is recapitulated from the tables in [36] with a different arrangement.

to identify the $\mathrm{u}(1)$ charge correctly and describe the reduced grand potential by the characters with the $\mathrm{u}(1)$ fugacity. From this viewpoint, in addition to the previous $\mathrm{u}(1)$ charge $d_{\mathrm{I}}$ appearing in decomposing the so(10) representations to so $(8) \times \mathrm{u}(1)$, we introduce another $\mathrm{u}(1)$ charge $d_{\mathrm{II}}$, both of which are given explicitly in the current degrees by

$$
d_{\mathrm{I}}=\left(d_{1}^{+}+d_{2}^{+}+d_{3}^{+}\right)-\left(d_{1}^{-}+d_{2}^{-}+d_{3}^{-}\right), \quad d_{\mathrm{II}}=\left(d_{1}^{+}-d_{1}^{-}\right)-\left(d_{3}^{+}-d_{3}^{-}\right) .
$$

With these two $\mathrm{u}(1)$ charges we can rearrange table 2 and table 3 in [36] by table 4 .

After the rearrangement it is not difficult to find the relation to the decomposition of the so(8) representations to $[\mathrm{su}(2)]^{4}$. Namely, due to the decomposition of the first few so(8) representations,

$$
\begin{aligned}
8_{\mathrm{v}} & \rightarrow(2,2,1,1)+(1,1,2,2), \\
8_{\mathrm{s}} & \rightarrow(2,1,2,1)+(1,2,1,2), \\
8_{\mathrm{c}} & \rightarrow(2,1,1,2)+(2,1,1,2), \\
28 & \rightarrow(3,1,1,1)+(1,3,1,1)+(1,1,3,1)+(1,1,1,3)+(2,2,2,2),
\end{aligned}
$$

we can successfully identify the $\mathrm{u}(1)$ charge as the Cartan subalgebra of the last $\mathrm{su}(2)$.

From this identification of the $\mathrm{u}(1)$ charge we can introduce another character with two parameters and describe the worldsheet and membrane instantons as

$$
J^{\mathrm{WS}}\left(\mu_{\mathrm{eff}}\right)=\sum_{m=1}^{\infty} d_{m} e^{-m \frac{\mu_{\mathrm{eff}}}{k}}, \quad J^{\mathrm{MB}}\left(\mu_{\mathrm{eff}}\right)=\sum_{\ell=1}^{\infty}\left(\widetilde{b}_{\ell} \mu_{\mathrm{eff}}+\widetilde{c}_{\ell}\right) e^{-\ell \mu_{\mathrm{eff}}}
$$

where the instanton coefficients are given by

$$
d_{m}=\sum_{n d=m} \frac{(-1)^{m}}{n} \sum_{j_{\mathrm{L}}, j_{\mathrm{R}}} n_{j_{\mathrm{L}}, j_{\mathrm{R}}}^{d, \mathbf{R}} \frac{(-1)^{d-1}}{\left(2 \sin \frac{\pi n}{k}\right)^{2}} \chi_{\mathbf{R}}\left(e^{-\pi i n b_{\mathrm{I}}}, e^{-\pi i n b_{\mathrm{II}}}\right) \chi_{j_{\mathrm{L}}}\left(e^{\frac{2 \pi n i}{k}}\right) \chi_{j_{\mathrm{R}}}(1),
$$




$$
\begin{aligned}
& \widetilde{b}_{\ell}=\sum_{n d=\ell} \frac{1}{n} \sum_{j_{\mathrm{L}}, j_{\mathrm{R}}} n_{j_{\mathrm{L}}, j_{\mathrm{R}}}^{d, \mathbf{R}} \frac{(-1)^{d} d}{4 \pi \sin \pi n k} \chi_{\mathbf{R}}\left(e^{-\pi i n k b_{\mathrm{I}}}, e^{-\pi i n k b_{\mathrm{II}}}\right) \chi_{j_{\mathrm{L}}}\left(e^{\pi i n k}\right) \chi_{j_{\mathrm{R}}}\left(e^{\pi i n k}\right), \\
& \widetilde{c}_{\ell}=-k^{2} \frac{\partial}{\partial k}\left[\frac{\widetilde{b}_{\ell}}{\ell k}\right]_{b_{\mathrm{I}}, b_{\mathrm{II}}},
\end{aligned}
$$

with $\left(b_{\mathrm{I}}, b_{\mathrm{II}}\right)=\left(1-M_{\mathrm{I}} / k,-2 M_{\mathrm{II}} / k\right)$. Note that in the coefficient $\widetilde{c}_{\ell}$ we treat $b_{\mathrm{I}}$ and $b_{\mathrm{II}}$ to be independent of $k$ under the derivative. Using the representations of so(10) in table 1 , table 2, table 3 and the characters in appendix A.4, we find that this simple expression reproduces all the instanton coefficients listed in appendix $\mathrm{C}$ of [36] for $1 \leq d \leq 8$.

\section{Orbifold $(2,1)$ model}

There is one more interesting theory which is solvable from the group-theoretical viewpoint. One lesson we learned from the study of the superconformal Chern-Simons theory with the orthosymplectic gauge group in [43] (see also [32, 33, 44, 45]) is that sometimes the duplicate quiver is easier than the original one. In the previous sections we have struggled for expressing the reduced grand potential of the $(2,1)$ model in terms of the free energy of topological strings (1.1). Here instead let us consider the duplicate $(2,1,2,1)$ model, which is the $\mathrm{U}(N)^{6}$ superconformal Chern-Simons theory with $\left\{s_{a}\right\}=\{+1,+1,-1,+1,+1,-1\}$. The physical interpretation of the repetition of $\left\{s_{a}\right\}$ is the orbifold $[22,23]$ and we often refer to the $(2,1,2,1)$ model also as the $\mathbb{Z}_{2}$ orbifold of the $(2,1)$ model. Since the odd membrane instantons of the $(2,1)$ model $(2.10)$ look very similar to those of the orthosymplectic theory [43], it is natural to expect that the odd membrane instantons are projected out in the duplicate $(2,1,2,1)$ model as well and the reduced grand potential falls into the standard expression (1.1) easily.

Before starting the computation of the instantons in the $(2,1,2,1)$ model, let us guess which set of the BPS indices should govern the model. From the Newton polygon, the general deformation of the $(2,1,2,1)$ model corresponds to a genus-three curve, which seems not so easy from the current technology. However, as explained carefully in [40, 46] (see figure 8 in [40]), the $E_{7}$ del Pezzo geometry also appears as a special case of the same curve with the parameters tuned (which reduces the curve to genus-one) ${ }^{7}$ Hence, we expect that the $(2,1,2,1)$ model is governed by the BPS indices of the local $E_{7}$ del Pezzo geometry.

\subsection{Instantons}

The reduced grand potential of the $\mathbb{Z}_{2}$ orbifold theory $J^{[2]}(\mu)$ can be obtained from the original one $J^{[1]}(\mu)$ by applying the rule of [47],

$$
\begin{aligned}
J^{[2]}(\mu)= & J^{[1]}\left(\frac{\mu+\pi i}{2}\right)+J^{[1]}\left(\frac{\mu-\pi i}{2}\right) \\
& +\log \left[1+\sum_{n \neq 0} e^{J^{[1]}\left(\frac{\mu+\pi i}{2}+2 \pi i n\right)+J^{[1]}\left(\frac{\mu-\pi i}{2}-2 \pi i n\right)-J^{[1]}\left(\frac{\mu+\pi i}{2}\right)-J^{[1]}\left(\frac{\mu-\pi i}{2}\right)}\right] .
\end{aligned}
$$

\footnotetext{
${ }^{7}$ We can check explicitly that, in the classical limit $k \rightarrow 0$, the genus of the curve degenerates due to the singularity of the curve. We thank Yasuhiko Yamada for valuable discussions.
} 
Since we shall discuss both the $(2,1)$ model and the $(2,1,2,1)$ model, to avoid confusions, we put the superscripts $(2,1)$ and $(2,1,2,1)$ to each quantity in this subsection to denote which model the quantity is associated to.

When we consider the duplicate model in (6.1), we need to substitute $(\mu \pm \pi i) / 2 \pm 2 \pi i n$ for $\mu$. The reduced grand potential $J^{(2,1)}(\mu)(2.4)$ depends on $\mu$ only through $\mu_{\text {eff }}^{(2,1)}$ in (2.6). Since the instanton effect in (2.6) is simply $e^{-2 \mu}$, we can define a common effective chemical potential $\mu_{\text {eff }}^{(2,1,2,1)}$ for the $(2,1,2,1)$ model

$$
\frac{\mu_{\text {eff }}^{(2,1,2,1)}}{2}= \begin{cases}\frac{\mu}{2}+2 e^{-\mu}{ }_{4} F_{3}\left(1,1, \frac{3}{2}, \frac{3}{2} ; 2,2,2 ;-16 e^{-\mu}\right), & \text { for odd } k, \\ \frac{\mu}{2}+6 e^{-\mu} F_{3}\left(1,1, \frac{7}{4}, \frac{5}{4} ; 2,2,2 ;-64 e^{-\mu}\right), & \text { for even } k,\end{cases}
$$

independent of $n$ and substitute $\left(\mu_{\mathrm{eff}}^{(2,1,2,1)} \pm \pi i\right) / 2 \pm 2 \pi i n$ for $\mu_{\mathrm{eff}}^{(2,1)}$ in (2.4).

Note that there is a great simplification in the "twisted" sectors $(n \neq 0)$ for the current case. After the substitution, the exponent in the twisted sector becomes

$$
\begin{aligned}
& J^{(2,1)}\left(\frac{\mu+\pi i}{2}+2 \pi i n\right)+J^{(2,1)}\left(\frac{\mu-\pi i}{2}-2 \pi i n\right)-J^{(2,1)}\left(\frac{\mu+\pi i}{2}\right)-J^{(2,1)}\left(\frac{\mu-\pi i}{2}\right) \\
& =-2 n(2 n+1) \pi^{2} C^{(2,1)} \mu_{\mathrm{eff}}^{(2,1,2,1)}-4 \sum_{m=1}^{\infty} d_{m}^{(2,1)} \sin \left(\frac{(2 n+1) m \pi}{k}\right) \sin \left(\frac{2 n m \pi}{k}\right) e^{-\frac{m}{k} \mu_{\mathrm{eff}}^{(2,1,2,1)}} .
\end{aligned}
$$

Note that both the even and odd membrane instanton parts cancel among themselves, leaving only the perturbative part and the worldsheet instanton part. Furthermore, if we use the result $C^{(2,1)}=1 /\left(\pi^{2} k\right)$, the exponential function of the perturbative part becomes $e^{-\frac{2 n(2 n+1)}{k} \mu_{\mathrm{eff}}^{(2,1,2,1)}}$, giving rise to the worldsheet instanton contribution. Namely, after substituting the worldsheet instanton part (6.3) into the logarithmic function in (6.1), we find that the twisted sectors only give the worldsheet instanton for the current case.

Therefore, the membrane instanton part comes directly from the "untwisted" sector $(n=0)$, as was the case for the perturbative part [47]. Again, the contributions from the odd membrane instantons $e^{-(2 \ell-1) \mu_{\text {eff }}^{(2,1)}}$ of the $(2,1)$ model disappear and the even membrane instantons $e^{-2 \ell \mu_{\text {eff }}^{(2,1)}}$ give rise to the standard expression of the membrane instantons $e^{-\ell \mu_{\mathrm{eff}}^{(2,1,2,1)}}$. Finally, we find that the reduced grand potential of the $(2,1,2,1)$ model becomes

$$
\begin{aligned}
J^{(2,1,2,1)}(\mu) & =J^{(2,1,2,1) \text { pert }}\left(\mu_{\mathrm{eff}}^{(2,1,2,1)}\right)+J^{(2,1,2,1) \mathrm{np}}\left(\mu_{\mathrm{eff}}^{(2,1,2,1)}\right), \\
J^{(2,1,2,1) \mathrm{np}}\left(\mu_{\mathrm{eff}}^{(2,1,2,1)}\right) & =J^{(2,1,2,1) \mathrm{WS}}\left(\mu_{\mathrm{eff}}^{(2,1,2,1)}\right)+J^{(2,1,2,1) \mathrm{MB}}\left(\mu_{\mathrm{eff}}^{(2,1,2,1)}\right),
\end{aligned}
$$

with each part given by

$$
\begin{aligned}
& J^{(2,1,2,1) \operatorname{pert}}\left(\mu_{\mathrm{eff}}^{(2,1,2,1)}\right)=\frac{C^{(2,1,2,1)}}{3}\left(\mu_{\mathrm{eff}}^{(2,1,2,1)}\right)^{3}+B^{(2,1,2,1)} \mu_{\mathrm{eff}}^{(2,1,2,1)}+A^{(2,1,2,1)}, \\
& J^{(2,1,2,1) \mathrm{WS}}\left(\mu_{\mathrm{eff}}^{(2,1,2,1)}\right)=\sum_{m=1}^{\infty} d_{m}^{(2,1,2,1)} e^{-\frac{m}{k} \mu_{\mathrm{eff}}^{(2,1,2,1)}} \\
& J^{(2,1,2,1) \mathrm{MB}}\left(\mu_{\mathrm{eff}}^{(2,1,2,1)}\right)=\sum_{\ell=1}^{\infty}\left(\widetilde{b}_{\ell}^{(2,1,2,1)} \mu_{\mathrm{eff}}^{(2,1,2,1)}+\widetilde{c}_{\ell}^{(2,1,2,1)}\right) e^{-\ell \mu_{\mathrm{eff}}^{(2,1,2,1)}} .
\end{aligned}
$$


Here the coefficients of the perturbative part are given by

$$
C^{(2,1,2,1)}=\frac{1}{4 \pi^{2} k}, \quad B^{(2,1,2,1)}=-\frac{1}{3 k}+\frac{k}{12},
$$

while those of the membrane instantons are given by

$$
\widetilde{b}_{\ell}^{(2,1,2,1)}=(-1)^{\ell} \widetilde{b}_{2 \ell}^{(2,1)}, \quad \widetilde{c}_{\ell}^{(2,1,2,1)}=2(-1)^{\ell} \widetilde{c}_{2 \ell}^{(2,1)},
$$

which indicates the derivative relation

$$
\widetilde{c}_{\ell}^{(2,1,2,1)}=-k^{2} \frac{d}{d k} \frac{\widetilde{b}_{\ell}^{(2,1,2,1)}}{\ell k} .
$$

The coefficients of the worldsheet instantons $d_{m}^{(2,1,2,1)}$ are obtained by multiplying $d_{m}^{(2,1)}$ with the cosine factor $2 \cos m \pi / k$ coming from the substitution of $\left(\mu_{\mathrm{eff}}^{(2,1,2,1)} \pm \pi i\right) / 2(6.2)$ and also taking into account the twisted sector (6.3) with $n$ satisfying $2 n(2 n+1) \leq m$. The explicit relations for the first few coefficients are given as

$$
\begin{aligned}
d_{1}^{(2,1,2,1)} & =2 \cos \left(\frac{\pi}{k}\right) d_{1}^{(2,1)}, \quad d_{2}^{(2,1,2,1)}=2 \cos \left(\frac{2 \pi}{k}\right) d_{2}^{(2,1)}+1 \\
d_{3}^{(2,1,2,1)} & =2 \cos \left(\frac{3 \pi}{k}\right) d_{3}^{(2,1)}-4 \sin \left(\frac{\pi}{k}\right) \sin \left(\frac{2 \pi}{k}\right) d_{1}^{(2,1)} \\
d_{4}^{(2,1,2,1)} & =2 \cos \left(\frac{4 \pi}{k}\right) d_{4}^{(2,1)}-4 \sin \left(\frac{2 \pi}{k}\right) \sin \left(\frac{4 \pi}{k}\right) d_{2}^{(2,1)}+8 \sin ^{2}\left(\frac{\pi}{k}\right) \sin ^{2}\left(\frac{2 \pi}{k}\right)\left(d_{1}^{(2,1)}\right)^{2}-\frac{1}{2}, \\
\cdots &
\end{aligned}
$$

To summarize, we obtain the membrane instanton coefficients in the $(2,1,2,1)$ model of degree $\ell$ directly from those in the $(2,1)$ model of degree $2 \ell$ using $(6.7)$, while for the worldsheet instanton coefficients of degree $m$ we need to expand (6.1) up to the $m$-th order with the help of (6.3) as in (6.9). Hereafter we shall only discuss the $(2,1,2,1)$ model and omit the superscript $(2,1,2,1)$.

\subsection{Characters}

Due to the difference in the odd instantons and the even instantons, we adopt an alternating multi-covering structure motivated by (4.15).

$$
d_{m}=\sum_{n \mid m} \frac{1}{n}\left(\delta_{\frac{m}{n}}^{+}\left(\frac{k}{n}\right)+(-1)^{n} \delta_{\frac{m}{n}}^{-}\left(\frac{k}{n}\right)\right) .
$$

By comparing with the result obtained in (6.1), we find that the first few instanton coefficients are given by

$$
\begin{aligned}
& \delta_{1}^{+}(k)-\delta_{1}^{-}(k)=\frac{8}{\left(2 \sin \frac{\pi}{k}\right)^{2}}, \quad \delta_{1}^{+}(k)+\delta_{1}^{-}(k)=\frac{56}{\left(2 \sin \frac{\pi}{k}\right)^{2}}, \\
& \delta_{2}^{+}(k)-\delta_{2}^{-}(k)=-\frac{16}{\left(2 \sin \frac{\pi}{k}\right)^{2}}+3,
\end{aligned}
$$


which gives

$$
\delta_{1}^{+}(k)=\frac{32}{\left(2 \sin \frac{\pi}{k}\right)^{2}}, \quad \delta_{1}^{-}(k)=\frac{24}{\left(2 \sin \frac{\pi}{k}\right)^{2}} .
$$

It is then interesting to compare these coefficients with the tables for the BPS indices of the local $E_{7}$ del Pezzo geometry in [37]. Let us decompose the $E_{7}$ representation 56 appearing in $d=1$ to the subalgebra $\mathrm{so}(12) \times \mathrm{su}(2)$,

$$
56 \rightarrow(12,2)+(32,1)
$$

and identify 32 and 24 in the numerator in (6.12) respectively as $32 \times 1$ and $12 \times 2$. We can imagine that the even(bosonic) and odd(fermionic) representations in the congruency class of $\mathrm{su}(2)$ contribute to $\delta_{d}^{+}(k)$ and $\delta_{d}^{-}(k)$ respectively.

Namely, once the BPS indices in the tables of [37] are partitioned into the $E_{7}$ representations

$$
N_{j_{\mathrm{L}}, j_{\mathrm{R}}}^{d}=(-1)^{d-1} \sum_{\mathbf{R}} n_{j_{\mathrm{L}}, j_{\mathrm{R}}}^{d, \mathbf{R}} \operatorname{dim}(\mathbf{R}),
$$

we propose that the multi-covering component of the worldsheet instantons is computed by

$$
\delta_{d}^{ \pm}(k)=\frac{(-1)^{d-1}}{\left(2 \sin \frac{\pi}{k}\right)^{2}} \sum_{j_{\mathrm{L}}, j_{\mathrm{R}}} \sum_{\mathbf{R}} n_{j_{\mathrm{L}}, j_{\mathrm{R}}}^{d, \mathbf{R}} n_{\mathbf{R}}^{ \pm} \chi_{j_{\mathrm{L}}}\left(e^{\frac{2 \pi i}{k}}\right) \chi_{j_{\mathrm{R}}}(1),
$$

where we have defined

$$
n_{\mathbf{R}}^{+/-}=\sum_{\rho: \text { even } / \text { odd }} \operatorname{dim} \boldsymbol{\rho} \cdot \operatorname{dim} \mathbf{r}
$$

for the decomposition

$$
E_{7} \rightarrow \mathrm{so}(12) \times \mathrm{su}(2), \quad \mathbf{R} \rightarrow \sum(\mathbf{r}, \boldsymbol{\rho}) .
$$

For the membrane instantons, we assume the standard multi-covering structure

$$
\widetilde{b}_{\ell}=\sum_{n \mid \ell} \frac{1}{n} \beta_{\frac{\ell}{n}}(n k),
$$

though for the comparison with the worldsheet instantons we also need the parity separation

$$
\beta_{d}(k)=\beta_{d}^{+}(k)+\beta_{d}^{-}(k), \quad \beta_{d}^{ \pm}(k)=\frac{(-1)^{d} d}{4 \pi \sin \pi k} \sum_{j_{\mathrm{L}}, j_{\mathrm{R}}} \sum_{\mathbf{R}} n_{j_{\mathrm{L}}, j_{\mathrm{R}}}^{d, \mathbf{R}} \chi_{\mathbf{R}}^{ \pm}\left(e^{-\pi i k}\right) \chi_{j_{\mathrm{L}}}\left(e^{\pi i k}\right) \chi_{j_{\mathrm{R}}}\left(e^{\pi i k}\right) .
$$

Here we have defined the $E_{7}$ characters $\chi_{\mathbf{R}}^{ \pm}(q)$ from the so(12) characters $\chi_{\mathbf{r}}(q)$ as

$$
\chi_{\mathbf{R}}^{ \pm}(q)=\sum_{\boldsymbol{\rho} \text { :even } / \text { odd }} \operatorname{dim} \boldsymbol{\rho} \cdot \chi_{\mathbf{r}}(q), \quad \chi_{\mathbf{r}}(q)=\sum_{h} q^{h} \operatorname{dim}\left(\mathbf{r}^{\prime}\right)_{h},
$$

with $h$ specifying the $\mathrm{u}(1)$ charge in the further decomposition of the so(12) representations to the subalgebra $\mathrm{so}(10) \times \mathrm{u}(1)$

$$
\mathrm{so}(12) \rightarrow \mathrm{so}(10) \times \mathrm{u}(1), \quad \mathbf{r} \rightarrow \sum\left(\mathbf{r}^{\prime}\right)_{h}
$$




\begin{tabular}{|c|c|c|c|}
\hline$d$ & $\left(j_{\mathrm{L}}, j_{\mathrm{R}}\right)$ & BPS & representations \\
\hline 1 & $(0,0)$ & 56 & 56 \\
\hline \multirow[t]{2}{*}{2} & $\left(0, \frac{1}{2}\right)$ & 133 & 133 \\
\hline & $\left(\frac{1}{2}, 1\right)$ & 1 & 1 \\
\hline \multirow[t]{2}{*}{3} & $(0,1)$ & 912 & 912 \\
\hline & $(0,0),\left(\frac{1}{2}, \frac{3}{2}\right)$ & 56 & 56 \\
\hline \multirow[t]{5}{*}{4} & $\left(0, \frac{3}{2}\right)$ & 8778 & $8645+133$ \\
\hline & $\left(0, \frac{1}{2}\right),\left(\frac{1}{2}, 2\right)$ & 1673 & $1539+133+1$ \\
\hline & $\left(\frac{1}{2}, 1\right)$ & 134 & $133+1$ \\
\hline & $\left(1, \frac{5}{2}\right)$ & 133 & 133 \\
\hline & $\left(0, \frac{5}{2}\right),\left(1, \frac{3}{2}\right),\left(\frac{3}{2}, 3\right)$ & 1 & 1 \\
\hline \multirow[t]{8}{*}{5} & $(0,2)$ & 93688 & $86184+6480+912+2 \times 56$ \\
\hline & $(0,1),\left(\frac{1}{2}, \frac{5}{2}\right)$ & 36080 & $\mathbf{2 7 6 6 4}+\mathbf{6 4 8 0}+2 \times \mathbf{9 1 2}+2 \times \mathbf{5 6}$ \\
\hline & $\left(\frac{1}{2}, \frac{3}{2}\right)$ & 8472 & $\mathbf{6 4 8 0}+2 \times \mathbf{9 1 2}+3 \times \mathbf{5 6}$ \\
\hline & $(1,3)$ & 7504 & $\mathbf{6 4 8 0}+\mathbf{9 1 2}+2 \times \mathbf{5 6}$ \\
\hline & $(0,0)$ & 6592 & $\mathbf{6 4 8 0}+2 \times 56$ \\
\hline & $(1,2)$ & 1024 & $\mathbf{9 1 2}+2 \times \mathbf{5 6}$ \\
\hline & $(0,3),\left(\frac{1}{2}, \frac{1}{2}\right),\left(\frac{3}{2}, \frac{7}{2}\right)$ & 968 & $912+56$ \\
\hline & $\left(\frac{1}{2}, \frac{7}{2}\right),(1,1),\left(\frac{3}{2}, \frac{5}{2}\right),(2,4)$ & 56 & 56 \\
\hline
\end{tabular}

Table 5. The constituent representations for the total BPS indices of the $(2,1,2,1)$ model for $1 \leq d \leq 5$.

With this identification, the remaining task is to separate the BPS indices given in [37] as the $E_{7}$ representations. Fortunately, this is given explicitly in [37] (see table 5). Surprisingly, we can confirm that the BPS indices with the identification of the representations given in [37] correctly reproduce the worldsheet instantons and the membrane instantons in appendix B. 1 for $1 \leq d \leq 4$ after decomposing the $E_{7}$ representations to so(10) $\times \mathrm{u}(1) \times \mathrm{su}(2)$ using the group-theoretical results in appendix B.2. Comparing the congruency class $\mathbb{Z}_{2}$ of $E_{7}$, it is interesting to observe that all of the representations appearing in degree $d$ belongs to the class $d$ mod 2 .

The identification of the representations for $d=5$ given in [37], however, does not obey the congruency class and the decomposition of the $E_{7}$ representations does not give the instanton effects correctly. Hence we assume general degeneracies $n_{j_{\mathrm{L}}, j_{\mathrm{R}}}^{d=5, \mathbf{R}}$ of the representations obeying the congruency class and solve the conditions to match the worldsheet instantons and the membrane instantons listed in appendix B.1. We have found a unique positive solution $\left\{n_{j_{\mathrm{L}}, j_{\mathrm{R}}}^{d=5, \mathbf{R}}\right\}$ given in table 5 . 
As in the case of the rank-deformed $(2,2)$ model studied in the previous section, we could introduce five Kähler parameters

$$
T_{n}=\frac{\mu_{\mathrm{eff}}}{k}+n \pi i, \quad(n=0, \pm 1, \pm 2) .
$$

There are again, however, not enough data to completely determine the split of the BPS indices. We have chosen alternatively to express our final result with the characters.

\section{Discussions}

We have revisited the grand potential of the $(2,1)$ model. We first observe that the worldsheet instantons of the $(2,1)$ model coincide exactly with that of the rank deformed $(2,2)$ model through the relation (3.2). This gives us a hint for the novel multi-covering structure of the membrane instantons (3.5). We also observe that the BPS indices for the $(2,2)$ model are those for the local $D_{5}$ del Pezzo geometry with the decomposition of the so(10) representations to the subalgebra $\mathrm{so}(8) \times \mathrm{u}(1)$. With these observations in mind, we are able to construct a framework to reproduce the multi-covering structure (2.8), (3.5), (2.12) and the derivative relations (2.11) for the reduced grand potential of the $(2,1)$ model using the topological string free energy by introducing the four Kähler parameters (4.2). After identifying the BPS indices, we discover that the BPS indices are those obtained by further decomposing the $\mathrm{so}(10)$ representations to $\mathrm{so}(6) \times \mathrm{u}(1) \times \mathrm{u}(1)$. We also explain that it is natural that the same set of the BPS indices is used for both the $(2,2)$ model and the $(2,1)$ model from the viewpoint of the Newton polygon.

We have continued to study the $(2,1,2,1)$ model, which is the $\mathbb{Z}_{2}$ orbifold of the $(2,1)$ model, and find that this time the BPS indices are those of the local $E_{7}$ del Pezzo geometry, with the $E_{7}$ representations decomposed to the subalgebra $\operatorname{so}(10) \times \mathrm{u}(1) \times \operatorname{su}(2)$. Though we have not been able to identify the correct representations for the local $E_{7}$ del Pezzo geometry in $d=6$ so far, we have listed the worldsheet instanton and the membrane instanton in appendix B.1 so that it can be checked in the future.

From the viewpoint of five-dimensional gauge theories [48], the local $D_{5}$ del Pezzo geometry and the local $E_{7}$ del Pezzo geometry are respectively associated to the $\mathcal{N}=1$ $\mathrm{SU}(2)$ Yang-Mills theories with $N_{f}=4$ and $N_{f}=6$ matters, possessing the perturbative flavor symmetries so(8) and so(12). It is only after we include the non-perturbative effects that the flavor symmetries are enhanced to $D_{5}$ and $E_{7}$. This may explain why we first consider the decomposition of the so(10) representations to so(8) $\times \mathrm{u}(1)$ and that of the $E_{7}$ representations to $\mathrm{so}(12) \times \mathrm{su}(2)$ when studying the instantons. Then, it remains to see which Weyl symmetries the models or the deformations preserve. It would be interesting to figure out the general rule to identify the $\mathrm{u}(1)$ charges.

In our determination of the representations, we have observed that the representations utilized in the BPS indices of degree $d$ are all in the congruency class $d$. We would like to know how this can be proved mathematically rigorously.

Years ago it was difficult to find the expression of the $(2,1)$ model and its cousins. We believe that our work has opened up a new avenue towards more general understanding of the partition function of the $\mathcal{N}=4$ superconformal Chern-Simons theories. We would 
like to pursue more examples, such as the $(p, q)$ models, for a concrete view of the nonperturbative effects.

From the above several examples along with those in [41, 42], the description of the nonperturbative effects of the reduced grand potential using the topological string theory (1.1) seems to work at least for the genus-one curve. For a general $(p, q)$ model the Newton polygon suggests the curve to be of higher genus, hence it is desired to know what the correct description is for higher genus curves. Especially we would like to see explicitly how recent proposals on the spectral determinant of higher genus curves $[49,50]$ works for these superconformal Chern-Simons theories. Our orbifold $(2,1)$ model may be instructive in the sense that on one hand the associated curve is generally of genus-three, though on the other hand the curve degenerates to genus-one.

\section{Acknowledgments}

We are grateful to Yasuyuki Hatsuda, Sung-Soo Kim, Kimyeong Lee, Takuya Matsumoto, Masatoshi Noumi, Soichi Okada, Kazumi Okuyama, Masato Taki, Akihiro Tsuchiya, Futoshi Yagi, Shintarou Yanagida and especially Yasuhiko Yamada for valuable discussions. We would like to thank Kobe university, department of mathematics for the kind hospitality where the final part of this work was performed. We are indebted to the authors of [41] for sharing their WKB data up to $\mathcal{O}\left(k^{29}\right)$ with us. The work of S.M. is supported by JSPS Grant-in-Aid for Scientific Research (C) \# 26400245.

\section{A Data for $(2,1) /(2,2)$ model and $D_{5}$}

In this appendix we summarize the data which are relevant in discussing the relation between the instanton effects of the $(2,1) /(2,2)$ models and the free energy of the topological string theory on the local $D_{5}$ del Pezzo geometry. In appendix A.1 we display the instanton coefficients of the $(2,1)$ model in terms of the multi-covering components. In appendix A.2 we list the irreducible representations of so(10) and the characters with a single $u(1)$ fugacity associated to the decomposition of the so(10) representations to the subalgebra $\mathrm{so}(8) \times \mathrm{u}(1)$. These data are used to determine the representations which the BPS indices of the local $D_{5}$ del Pezzo geometry consist of from the instanton coefficients of the $(2,2)$ model. In appendix A.3 we list the characters with an additional $\mathrm{u}(1)$ fugacity associated with the further decomposition of the so(8) representations to the subalgebra so(6) $\times \mathrm{u}(1)$, which appear in the instanton coefficients of the $(2,1)$ model. Finally, in appendix A.4 we turn on the second fugacity in a different way so that the characters reproduce the instanton coefficients of the $(2,2)$ model with the gauge group $\mathrm{U}\left(N+M_{\mathrm{II}}\right)_{k} \times \mathrm{U}\left(N+M_{\mathrm{I}}\right)_{0} \times \mathrm{U}\left(N+2 M_{\mathrm{I}}+M_{\mathrm{II}}\right)_{-k} \times \mathrm{U}\left(N+M_{\mathrm{I}}\right)_{0}$.

\section{A.1 Instanton coefficients for $(2,1)$ model}

We shall list the explicit form of the instanton coefficients for the $(2,1)$ model. The first several coefficients of the worldsheet instantons are given by

$$
\delta_{1}(k)=\frac{4 \cos \frac{\pi}{k}}{\sin ^{2} \frac{2 \pi}{k}}
$$




$$
\begin{aligned}
\delta_{2}(k)= & -\frac{4+\cos \frac{2 \pi}{k}}{\sin ^{2} \frac{2 \pi}{k}}, \\
\delta_{3}(k)= & \frac{12 \cos \frac{\pi}{k}}{\sin ^{2} \frac{2 \pi}{k}} \\
\delta_{4}(k)= & -\frac{32+16 \cos \frac{2 \pi}{k}}{\sin ^{2} \frac{2 \pi}{k}}+5 \\
\delta_{5}(k)= & \frac{220 \cos \frac{\pi}{k}+20 \cos \frac{3 \pi}{k}}{\sin ^{2} \frac{2 \pi}{k}}-96 \cos \frac{\pi}{k}, \\
\delta_{6}(k)= & -\frac{780+579 \cos \frac{2 \pi}{k}}{\sin ^{2} \frac{2 \pi}{k}}+\left(848+480 \cos \frac{2 \pi}{k}\right)-\left(256+64 \cos \frac{2 \pi}{k}\right) \sin ^{2} \frac{2 \pi}{k}, \\
\delta_{7}(k)= & \frac{7168 \cos \frac{\pi}{k}+1260 \cos \frac{3 \pi}{k}}{\sin ^{2} \frac{2 \pi}{k}}-\left(13232 \cos \frac{\pi}{k}+1696 \cos \frac{3 \pi}{k}\right) \\
& +\left(9472 \cos \frac{\pi}{k}+576 \cos \frac{3 \pi}{k}\right) \sin ^{2} \frac{2 \pi}{k}-2560 \cos \frac{\pi}{k} \sin ^{4} \frac{2 \pi}{k}
\end{aligned}
$$

while the odd membrane instantons are

$$
\begin{aligned}
\gamma_{1}(k)= & -\frac{\sin \pi k}{\sin ^{2} \frac{\pi k}{2}}, \\
\gamma_{3}(k)= & -\frac{\sin \pi k+\sin 2 \pi k}{\sin ^{2} \frac{\pi k}{2}}, \\
\gamma_{5}(k)= & -\frac{2 \sin \pi k+6 \sin 2 \pi k+6 \sin 3 \pi k+2 \sin 4 \pi k}{\sin ^{2} \frac{\pi k}{2}}, \\
\gamma_{7}(k)= & -(13 \sin \pi k+38 \sin 2 \pi k+68 \sin 3 \pi k+68 \sin 4 \pi k+38 \sin 5 \pi k+13 \sin 6 \pi k \\
& +2 \sin 7 \pi k) /\left(\sin ^{2} \frac{\pi k}{2}\right), \\
\gamma_{9}(k)= & -(150 \sin \pi k+397 \sin 2 \pi k+754 \sin 3 \pi k+1053 \sin 4 \pi k+1053 \sin 5 \pi k \\
& +754 \sin 6 \pi k+399 \sin 7 \pi k+164 \sin 8 \pi k+52 \sin 9 \pi k+14 \sin 10 \pi k+2 \sin 11 \pi k) \\
& /\left(\sin 2 \frac{\pi k}{2}\right), \\
\gamma_{11}(k)= & -(2469 \sin \pi k+5880 \sin 2 \pi k+10694 \sin 3 \pi k+16180 \sin 4 \pi k+20090 \sin 5 \pi k \\
& +20092 \sin 6 \pi k+16194 \sin 7 \pi k+10751 \sin 8 \pi k+6064 \sin 9 \pi k+3002 \sin 10 \pi k \\
& +1328 \sin 11 \pi k+533 \sin 12 \pi k+184 \sin 13 \pi k+57 \sin 14 \pi k+14 \sin 15 \pi k \\
& +2 \sin 16 \pi k) /\left(\sin ^{2} \frac{\pi k}{2}\right),
\end{aligned}
$$

and the even membrane instantons are

$$
\begin{aligned}
& \beta_{2}^{\prime}(k)=\frac{4 \sin \pi k+\sin 2 \pi k}{2 \pi \sin ^{2} \frac{\pi k}{2}}, \\
& \beta_{4}^{\prime}(k)=\frac{5 \sin \pi k+6 \sin 2 \pi k+5 \sin 3 \pi k}{\pi \sin ^{2} \frac{\pi k}{2}},
\end{aligned}
$$




$$
\begin{aligned}
\beta_{6}^{\prime}(k)= & \frac{3(14 \sin \pi k+28 \sin 2 \pi k+48 \sin 3 \pi k+28 \sin 4 \pi k+14 \sin 5 \pi k+\sin 6 \pi k)}{2 \pi \sin ^{2} \frac{\pi k}{2}}, \\
\beta_{8}^{\prime}(k)= & 4(43 \sin \pi k+98 \sin 2 \pi k+192 \sin 3 \pi k+214 \sin 4 \pi k+192 \sin 5 \pi k+98 \sin 6 \pi k \\
& +46 \sin 7 \pi k+10 \sin 8 \pi k+3 \sin 9 \pi k) /\left(\pi \sin ^{2} \frac{\pi k}{2}\right), \\
\beta_{10}^{\prime}(k)= & 5(904 \sin \pi k+2080 \sin 2 \pi k+3892 \sin 3 \pi k+5416 \sin 4 \pi k+6328 \sin 5 \pi k \\
& +5417 \sin 6 \pi k+3906 \sin 7 \pi k+2119 \sin 8 \pi k+1068 \sin 9 \pi k+400 \sin 10 \pi k \\
& +164 \sin 11 \pi k+39 \sin 12 \pi k+14 \sin 13 \pi k+\sin 14 \pi k) /\left(2 \pi \sin ^{2} \frac{\pi k}{2}\right), \\
\beta_{12}^{\prime}(k)= & 3(13269 \sin \pi k+29510 \sin 2 \pi k+51947 \sin 3 \pi k+76500 \sin 4 \pi k+99103 \sin 5 \pi k \\
+ & 106846 \sin 6 \pi k+99191 \sin 7 \pi k+76740 \sin 8 \pi k+52699 \sin 9 \pi k+31238 \sin 10 \pi k \\
& +17459 \sin 11 \pi k+8580 \sin 12 \pi k+4190 \sin 13 \pi k+1728 \sin 14 \pi k+752 \sin 15 \pi k \\
& +240 \sin 16 \pi k+94 \sin 17 \pi k+20 \sin 18 \pi k+6 \sin 19 \pi k) /\left(\pi \sin ^{2} \frac{\pi k}{2}\right) . \quad(\text { A.3 })
\end{aligned}
$$

The auxiliary membrane instantons borrowed from the $(2,2)$ model by replacing $k$ by $k / 2(3.7)$ are given as

$$
\begin{aligned}
& \beta_{1}(k)=-\frac{2 \sin \pi k}{\pi \sin ^{2} \frac{\pi k}{2}} \\
& \beta_{2}(k)=\frac{8 \sin \pi k+\sin 2 \pi k}{2 \pi \sin ^{2} \frac{\pi k}{2}}, \\
& \beta_{3}(k)=-\frac{6 \sin \pi k+6 \sin 2 \pi k}{\pi \sin ^{2} \frac{\pi k}{2}} \\
& \beta_{4}(k)=\frac{9 \sin \pi k+30 \sin 2 \pi k+9 \sin 3 \pi k}{\pi \sin ^{2} \frac{\pi k}{2}} \\
& \beta_{5}(k)=-\frac{20 \sin \pi k+100 \sin 2 \pi k+100 \sin 3 \pi k+20 \sin 4 \pi k}{\pi \sin ^{2} \frac{\pi k}{2}} .
\end{aligned}
$$

For higher instantons of the $(2,2)$ model, the function expression was not obtained from the WKB expansion

$$
\begin{aligned}
\beta_{6}^{(2,2)}(k)= & \frac{8146}{\pi^{2} k}-60732 k+\frac{835836 \pi^{2} k^{3}}{5}-\frac{26743288 \pi^{4} k^{5}}{105}+\frac{18972788 \pi^{6} k^{7}}{75}+\mathcal{O}\left(k^{9}\right), \\
\beta_{7}^{(2,2)}(k)= & -\frac{2890808}{49 \pi^{2} k}+\frac{1853576 k}{3}-\frac{110179048 \pi^{2} k^{3}}{45}+\frac{741506416 \pi^{4} k^{5}}{135}-\frac{5548809784 \pi^{6} k^{7}}{675} \\
& +\mathcal{O}\left(k^{9}\right), \\
\beta_{8}^{(2,2)}(k)= & \frac{7168777}{16 \pi^{2} k}-\frac{18917506 k}{3}+\frac{1543348448 \pi^{2} k^{3}}{45}-\frac{14523693056 \pi^{4} k^{5}}{135} \\
& +\frac{1083571808768 \pi^{6} k^{7}}{4725}+\mathcal{O}\left(k^{9}\right) .
\end{aligned}
$$

\section{A.2 Decomposition of so(10) representations}

In this appendix we list the decompositions of the first several so(10) irreducible representations to the subalgebra $s o(8) \times u(1)$. These decompositions are helpful in identifying the 


\begin{tabular}{|c|c|c|c|c|c|}
\hline $\mathrm{so}(10)$ & 0 & \pm 1 & \pm 2 & \pm 3 & \pm 4 \\
\hline $\mathbf{1}$ & $\mathbf{1}$ & & & & \\
\hline $\mathbf{1 0}$ & $\mathbf{8}_{\mathrm{v}}$ & & $\mathbf{1}$ & & \\
\hline $\mathbf{1 6}$ & & $\mathbf{8}_{\mathbf{s} / \mathbf{c}}$ & & & \\
\hline $\mathbf{4 5}$ & $\mathbf{2 8}+\mathbf{1}$ & & $\mathbf{8}_{\mathbf{v}}$ & & \\
\hline $\mathbf{5 4}$ & $\mathbf{3 5}+\mathbf{1}$ & & $\mathbf{8}_{\mathbf{v}}$ & & $\mathbf{1}$ \\
\hline $\mathbf{1 2 0}$ & $\mathbf{5 6} \mathbf{6}_{\mathbf{v}}+\mathbf{8}_{\mathbf{v}}$ & & $\mathbf{2 8}$ & & \\
\hline $\mathbf{1 2 6}$ & $\mathbf{5 6}$ & & $\mathbf{3 5}$ & & \\
\hline $\mathbf{1 4 4}$ & & $\mathbf{5 6} \mathbf{6}_{\mathrm{s} / \mathbf{c}}+\mathbf{8}_{\mathrm{s} / \mathbf{c}}$ & & $\mathbf{8}_{\mathrm{s} / \mathbf{c}}$ & \\
\hline
\end{tabular}

Table 6. The decomposition of the so(10) representations to the subalgebra so(8) $\times u(1)$.

irreducible representations which the total BPS indices listed in [37] consist of. We only list the first few representations necessary for the study of $1 \leq d \leq 5$ in table 6 . For higher degrees, we present the characters. The character for a general representation $\mathbf{R}$ of Lie algebra $g$ with fugacities $\xi$ can be computed by the Weyl character formula

$$
\chi_{\mathbf{R}}(\xi)=\lim _{\epsilon \rightarrow 0} \frac{\sum_{w \in W_{g}}(-1)^{l(w)} e^{\left(\xi+\epsilon \rho, w\left(d_{i} \omega_{i}+\rho\right)\right)}}{\sum_{w \in W_{g}}(-1)^{l(w)} e^{(\xi+\epsilon \rho, w(\rho))}} .
$$

Here $W_{g}$ is the Weyl group, $l(w)$ is the length of reflection $w \in W_{g}$ and $d_{i}$ is the Dynkin label of the representation $\mathbf{R}$ with $\omega_{i}$ being the fundamental weights and $\rho=\sum_{i} \omega_{i}$ being the Weyl vector. For the current case of the algebra so(10), if we choose the fundamental weights as

$$
\begin{array}{lll}
\omega_{1}=(1,0,0,0,0), & \omega_{2}=(1,1,0,0,0), & \omega_{3}=(1,1,1,0,0), \\
\omega_{4}=\left(\frac{1}{2}, \frac{1}{2}, \frac{1}{2}, \frac{1}{2},-\frac{1}{2}\right), & \omega_{5}=\left(\frac{1}{2}, \frac{1}{2}, \frac{1}{2}, \frac{1}{2}, \frac{1}{2}\right), &
\end{array}
$$

the fugacity for the $\mathrm{u}(1)$ charge in decomposing the so(10) representations to the subalgebra $\operatorname{so}(8) \times u(1)$ is $\xi=(2 \log q, 0,0,0,0)$.

The explicit expression of the characters are given by

$$
\begin{aligned}
\chi_{\mathbf{1}}(q) & =1 \\
\chi_{\mathbf{4 5}}(q) & =29+8\left(q^{2}+q^{-2}\right), \\
\chi_{\mathbf{5 4}}(q) & =36+8\left(q^{2}+q^{-2}\right)+q^{4}+q^{-4} \\
\chi_{\mathbf{2 1 0}}(q) & =98+56\left(q^{2}+q^{-2}\right) \\
\chi_{\mathbf{6 6 0}}(q) & =330+120\left(q^{2}+q^{-2}\right)+36\left(q^{4}+q^{-4}\right)+8\left(q^{6}+q^{-6}\right)+q^{8}+q^{-8}, \\
\chi_{\mathbf{7 7 0}}(q) & =364+168\left(q^{2}+q^{-2}\right)+35\left(q^{4}+q^{-4}\right), \\
\chi_{\mathbf{9 4 5}}(q) & =441+224\left(q^{2}+q^{-2}\right)+28\left(q^{4}+q^{-4}\right), \\
\chi_{\mathbf{1 0 5 0}}(q) & =420+280\left(q^{2}+q^{-2}\right)+35\left(q^{4}+q^{-4}\right),
\end{aligned}
$$




$$
\begin{aligned}
& \chi_{\mathbf{1 3 8 6}}(q)=666+288\left(q^{2}+q^{-2}\right)+64\left(q^{4}+q^{-4}\right)+8\left(q^{6}+q^{-6}\right), \\
& \chi_{\mathbf{2 7 7 2}}(q)=840+672\left(q^{2}+q^{-2}\right)+294\left(q^{4}+q^{-4}\right), \\
& \chi_{\mathbf{4 1 2 5}}(q)=1525+1000\left(q^{2}+q^{-2}\right)+300\left(q^{4}+q^{-4}\right),
\end{aligned}
$$

for the congruency class $d \equiv 0 \bmod 4$,

$$
\begin{aligned}
\chi_{\mathbf{1 0}}(q) & =8+q^{2}+q^{-2}, \\
\chi_{\mathbf{1 2 0}}(q) & =64+28\left(q^{2}+q^{-2}\right), \\
\chi_{\mathbf{1 2 6}}(q) & =56+35\left(q^{2}+q^{-2}\right), \\
\chi_{\mathbf{2 1 0}}(q) & =120+36\left(q^{2}+q^{-2}\right)+8\left(q^{4}+q^{-4}\right)+q^{6}+q^{-6}, \\
\chi_{\mathbf{3 2 0}}(q) & =176+64\left(q^{2}+q^{-2}\right)+8\left(q^{4}+q^{-4}\right),
\end{aligned}
$$

for the congruency class $d \equiv 2 \bmod 4$ and

$$
\begin{aligned}
\chi_{\mathbf{1 6}}(q) & =8\left(q+q^{-1}\right), \\
\chi_{\mathbf{1 4 4}}(q) & =64\left(q+q^{-1}\right)+8\left(q^{3}+q^{-3}\right), \\
\chi_{\mathbf{5 6 0}}(q) & =224\left(q+q^{-1}\right)+56\left(q^{3}+q^{-3}\right), \\
\chi_{\mathbf{6 7 2}}(q) & =224\left(q+q^{-1}\right)+112\left(q^{3}+q^{-3}\right), \\
\chi_{\mathbf{7 2 0}}(q) & =288\left(q+q^{-1}\right)+64\left(q^{3}+q^{-3}\right)+8\left(q^{5}+q^{-5}\right), \\
\chi_{\mathbf{1 2 0 0}}(q) & =440\left(q+q^{-1}\right)+160\left(q^{3}+q^{-3}\right), \\
\chi_{\mathbf{1 4 4 0}}(q) & =496\left(q+q^{-1}\right)+224\left(q^{3}+q^{-3}\right),
\end{aligned}
$$

for the congruency class $d \equiv 1,3 \bmod 4$.

\section{A.3 BPS indices for so(10) representations}

In the main text we have conjectured that the BPS indices appearing in the $(2,1)$ model are those obtained by decomposing the so(10) representations to the subalgebra so(6) $\times \mathrm{u}(1) \times \mathrm{u}(1)$. Then only a few combinations of the original BPS indices $N_{j_{\mathrm{L}}, j_{\mathrm{R}}}^{\left(d, d_{\mathrm{m}}, d_{\mathrm{m}}\right)}$, called the alternating BPS indices $N_{j_{\mathrm{L}}, j_{\mathrm{R}}}^{\prime\left(d, d_{\mathrm{m}}\right)}(4.15)$ and the weighted BPS indices $M_{j_{\mathrm{L}}, j_{\mathrm{R}}}^{\left(d, d_{\mathrm{m}}\right)}(4.19)$ appear in the membrane instanton of the $(2,1)$ model. Hence, in this appendix, we shall compute these indices for various so(10) representations.

These BPS indices can be computed from the characters with two fugacities indicating the two $\mathrm{u}(1)$ charges in decomposing the so(10) representations to the subalgebra so $(6) \times \mathrm{u}(1) \times \mathrm{u}(1)$. With the same choice of the fundamental weights (A.7), the characters can be obtained by substituting $\xi=(2 \log q, 2 \log p, 0,0,0)$ into (A.6), where we omit the characters of some so(10) representations which are not used for the BPS indices. Then, using (4.31), the two combinations of the BPS indices are obtained in table 7 from the characters.

The characters are given by

$$
\begin{aligned}
\chi_{\mathbf{1}}(p, q) & =1, \\
\chi_{\mathbf{4 5}}(p, q) & =17+6\left(q^{2}+q^{-2}\right)+\left(p^{2}+p^{-2}\right)\left(6+q^{2}+q^{-2}\right),
\end{aligned}
$$




\begin{tabular}{|c|c|c|c|c|c|c|c|c|c|c|c|c|}
\hline $\begin{array}{c}N_{j_{\mathrm{L}}, j_{\mathrm{R}}}^{\prime\left(d, d_{\mathrm{m}}\right)} \\
\end{array}$ & 0 & \pm 2 & \pm 4 & \pm 6 & & & & & & & & \\
\hline 1 & 1 & & & & & & & & & & & \\
\hline 45 & 5 & 4 & & & $N_{j_{\mathrm{L}}, j_{\mathrm{R}}}^{\prime\left(d, d_{\mathrm{m}}\right)}$ & 0 & \pm 2 & \pm 4 & $M_{j_{\mathrm{L}}, j_{\mathrm{R}}}^{\left(d, d_{\mathrm{m}}\right)}$ & \pm 1 & \pm 3 & \pm 5 \\
\hline 54 & 12 & 4 & 1 & & 10 & 4 & 1 & & 16 & 8 & & \\
\hline 210 & -6 & -4 & & & 120 & 0 & 4 & & 144 & 32 & 8 & \\
\hline 770 & 36 & 20 & 11 & & 126 & -4 & -5 & & 560 & 32 & 24 & \\
\hline 945 & 9 & 16 & 4 & & 320 & 24 & 16 & 4 & 720 & 96 & 32 & 8 \\
\hline 1050 & -20 & -20 & -5 & & & & & & & & & \\
\hline 1386 & 66 & 48 & 16 & 4 & & & & & & & & \\
\hline
\end{tabular}

Table 7. (Left two) The alternating BPS indices $(-1)^{d-1} N_{j_{\mathrm{L}}, j_{\mathrm{R}}}^{\left(d, d_{\mathrm{m}}\right)}$ for the representations of $\mathrm{so}(10)$ in the congruency class $d \equiv 0$ or $d \equiv 2 \bmod 4$ which are used in the membrane instanton effects $\beta_{d}^{\prime}(k)$ for $d=2,4,6,8$. (Right one) The weighted BPS indices $(-1)^{d-1} M_{j_{\mathrm{L}}, j_{\mathrm{R}}}^{\left(d, d_{\mathrm{m}}\right)}$ for the representations of so(10) in the congruency class $d \equiv 1$ or $d \equiv 3 \bmod 4$ which are used in the membrane instanton effects $\gamma_{d}(k)$ for $d=1,3,5,7$.

$$
\begin{aligned}
\chi_{\mathbf{5 4}}(p, q)= & 22+6\left(q^{2}+q^{-2}\right)+q^{4}+q^{-4}+\left(p^{2}+p^{-2}\right)\left(6+q^{2}+q^{-2}\right)+p^{4}+p^{-4}, \\
\chi_{\mathbf{2 1 0}}(p, q)= & 46+26\left(q^{2}+q^{-2}\right)+\left(p^{2}+p^{-2}\right)\left(26+15\left(q^{2}+q^{-2}\right)\right), \\
\chi_{\mathbf{7 7 0}}(p, q)= & 158+82\left(q^{2}+q^{-2}\right)+21\left(q^{4}+q^{-4}\right)+\left(p^{2}+p^{-2}\right)\left(82+37\left(q^{2}+q^{-2}\right)+6\left(q^{4}+q^{-4}\right)\right) \\
& +\left(p^{4}+p^{-4}\right)\left(21+6\left(q^{2}+q^{-2}\right)+q^{4}+q^{-4}\right), \\
\chi_{\mathbf{9 4 5}}(p, q)= & 193+108\left(q^{2}+q^{-2}\right)+16\left(q^{4}+q^{-4}\right) \\
& +\left(p^{2}+p^{-2}\right)\left(108+52\left(q^{2}+q^{-2}\right)+6\left(q^{4}+q^{-4}\right)\right)+\left(p^{4}+p^{-4}\right)\left(16+6\left(q^{2}+q^{-2}\right)\right), \\
\chi_{\mathbf{1 0 5 0}}(p, q)= & 170+110\left(q^{2}+q^{-2}\right)+15\left(q^{4}+q^{-4}\right) \\
& +\left(p^{2}+p^{-2}\right)\left(110+75\left(q^{2}+q^{-2}\right)+10\left(q^{4}+q^{-4}\right)\right)+\left(p^{4}+p^{-4}\right)\left(15+10\left(q^{2}+q^{-2}\right)\right), \\
\chi_{\mathbf{1 3 8 6}}(p, q)= & 290+144\left(q^{2}+q^{-2}\right)+38\left(q^{4}+q^{-4}\right)+6\left(q^{6}+q^{-6}\right) \\
& +\left(p^{2}+p^{-2}\right)\left(144+59\left(q^{2}+q^{-2}\right)+12\left(q^{4}+q^{-4}\right)+q^{6}+q^{-6}\right) \\
& +\left(p^{4}+p^{-4}\right)\left(38+12\left(q^{2}+q^{-2}\right)+q^{4}+q^{-4}\right)+\left(p^{6}+p^{-6}\right)\left(6+q^{2}+q^{-2}\right),
\end{aligned}
$$

for the congruency class $d \equiv 0 \bmod 4$,

$$
\begin{aligned}
\chi_{\mathbf{1 0}}(p, q)= & 6+q^{2}+q^{-2}+p^{2}+p^{-2}, \\
\chi_{\mathbf{1 2 0}}(p, q)= & 32+16\left(q^{2}+q^{-2}\right)+\left(p^{2}+p^{-2}\right)\left(16+6\left(q^{2}+q^{-2}\right)\right), \\
\chi_{\mathbf{1 2 6}}(p, q)= & 26+15\left(q^{2}+q^{-2}\right)+\left(p^{2}+p^{-2}\right)\left(15+10\left(q^{2}+q^{-2}\right)\right), \\
\chi_{\mathbf{3 2 0}}(p, q)= & 88+38\left(q^{2}+q^{-2}\right)+6\left(q^{4}+q^{-4}\right)+\left(p^{2}+p^{-2}\right)\left(38+12\left(q^{2}+q^{-2}\right)+q^{4}+q^{-4}\right) \\
& +\left(p^{4}+p^{-4}\right)\left(6+q^{2}+q^{-2}\right),
\end{aligned}
$$

for the congruency class $d \equiv 2 \bmod 4$ and

$$
\begin{aligned}
\chi_{\mathbf{1 6}}(p, q) & =4\left(p+p^{-1}\right)\left(q+q^{-1}\right), \\
\chi_{\mathbf{1 4 4}}(p, q) & =4\left(p+p^{-1}\right)\left(q+q^{-1}\right)\left[5+q^{2}+q^{-2}+p^{2}+p^{-2}\right],
\end{aligned}
$$




$$
\begin{aligned}
\chi_{\mathbf{5 6 0}}(p, q)= & 4\left(p+p^{-1}\right)\left(q+q^{-1}\right)\left[11+5\left(q^{2}+q^{-2}\right)+\left(p^{2}+p^{-2}\right)\left(5+q^{2}+q^{-2}\right)\right] \\
\chi_{\mathbf{7 2 0}}(p, q)= & 4\left(p+p^{-1}\right)\left(q+q^{-1}\right)\left[17+5\left(q^{2}+q^{-2}\right)+q^{4}+q^{-4}\right. \\
& \left.+\left(p^{2}+p^{-2}\right)\left(5+q^{2}+q^{-2}\right)+p^{4}+p^{-4}\right]
\end{aligned}
$$

for the congruency class $d \equiv 1,3 \bmod 4$.

\section{A.4 Characters for rank-deformed $(2,2)$ model}

In this appendix we shall list the so(10) characters with two parameters for the study of the $(2,2)$ model with the rank deformation $\mathrm{U}\left(N+M_{\mathrm{II}}\right)_{k} \times \mathrm{U}(N+$ $\left.M_{\mathrm{I}}\right)_{0} \times \mathrm{U}\left(N+2 M_{\mathrm{I}}+M_{\mathrm{II}}\right)_{-k} \times \mathrm{U}\left(N+M_{\mathrm{I}}\right)_{0}$. The characters are obtained by setting $\xi=\left(2 \log q_{\mathrm{I}}, \log q_{\mathrm{II}}, \log q_{\mathrm{II}}, 0,0\right)$ in the Weyl character formula (A.6) for the same choice of the fundamental weights (A.7). The characters are given explicitly by

$$
\begin{aligned}
\chi_{\mathbf{1}}\left(q_{\mathrm{I}}, q_{\mathrm{II}}\right)= & 1, \\
\chi_{\mathbf{4 5}}\left(q_{\mathrm{I}}, q_{\mathrm{II}}\right)= & 11+8\left(q_{\mathrm{II}}+q_{\mathrm{II}}^{-1}\right)+q_{\mathrm{II}}^{2}+q_{\mathrm{II}}^{-2}+\left(q_{\mathrm{I}}^{2}+q_{\mathrm{I}}^{-2}\right)\left(4+2\left(q_{\mathrm{II}}+q_{\mathrm{II}}^{-1}\right)\right), \\
\chi_{\mathbf{5 4}}\left(q_{\mathrm{I}}, q_{\mathrm{II}}\right)= & 14+8\left(q_{\mathrm{II}}+q_{\mathrm{II}}^{-1}\right)+3\left(q_{\mathrm{II}}^{2}+q_{\mathrm{II}}^{-2}\right)+\left(q_{\mathrm{I}}^{2}+q_{\mathrm{I}}^{-2}\right)\left(4+2\left(q_{\mathrm{II}}+q_{\mathrm{II}}^{-1}\right)\right)+q_{\mathrm{I}}^{4}+q_{\mathrm{I}}^{-4}, \\
\chi_{\mathbf{2 1 0}}\left(q_{\mathrm{I}}, q_{\mathrm{II}}\right)= & 36+24\left(q_{\mathrm{II}}+q_{\mathrm{II}}^{-1}\right)+7\left(q_{\mathrm{II}}^{2}+q_{\mathrm{II}}^{-2}\right) \\
& +\left(q_{\mathrm{I}}^{2}+q_{\mathrm{I}}^{-2}\right)\left(20+14\left(q_{\mathrm{II}}+q_{\mathrm{II}}^{-1}\right)+4\left(q_{\mathrm{II}}^{2}+q_{\mathrm{II}}^{-2}\right)\right), \\
\chi_{\mathbf{7 7 0}}\left(q_{\mathrm{I}}, q_{\mathrm{II}}\right)= & 104+80\left(q_{\mathrm{II}}+q_{\mathrm{II}}^{-1}\right)+41\left(q_{\mathrm{II}}^{2}+q_{\mathrm{II}}^{-2}\right)+8\left(q_{\mathrm{II}}^{3}+q_{\mathrm{II}}^{-3}\right)+q_{\mathrm{II}}^{4}+q_{\mathrm{II}}^{-4} \\
& +\left(q_{\mathrm{I}}^{2}+q_{\mathrm{I}}^{-2}\right)\left(52+40\left(q_{\mathrm{II}}+q_{\mathrm{II}}^{-1}\right)+16\left(q_{\mathrm{II}}^{2}+q_{\mathrm{II}}^{-2}\right)+2\left(q_{\mathrm{II}}^{3}+q_{\mathrm{II}}^{-3}\right)\right) \\
& +\left(q_{\mathrm{I}}^{4}+q_{\mathrm{I}}^{-4}\right)\left(13+8\left(q_{\mathrm{II}}+q_{\mathrm{II}}^{-1}\right)+3\left(q_{\mathrm{II}}^{2}+q_{\mathrm{II}}^{-2}\right)\right), \\
\chi_{\mathbf{9 4 5}}\left(q_{\mathrm{I}}, q_{\mathrm{II}}\right)= & 133+104\left(q_{\mathrm{II}}+q_{\mathrm{II}}^{-1}\right)+42\left(q_{\mathrm{II}}^{2}+q_{\mathrm{II}}^{-2}\right)+8\left(q_{\mathrm{II}}^{3}+q_{\mathrm{II}}^{-3}\right) \\
& +\left(q_{\mathrm{I}}^{2}+q_{\mathrm{I}}^{-2}\right)\left(72+54\left(q_{\mathrm{II}}+q_{\mathrm{II}}^{-1}\right)+20\left(q_{\mathrm{II}}^{2}+q_{\mathrm{II}}^{-2}\right)+2\left(q_{\mathrm{II}}^{3}+q_{\mathrm{II}}^{-3}\right)\right) \\
& +\left(q_{\mathrm{I}}^{4}+q_{\mathrm{I}}^{-4}\right)\left(10+8\left(q_{\mathrm{II}}+q_{\mathrm{II}}^{-1}\right)+q_{\mathrm{II}}^{2}+q_{\mathrm{II}}^{-2}\right), \\
\chi_{\mathbf{1 0 5 0}}\left(q_{\mathrm{I}}, q_{\mathrm{II}}\right)= & 126+96\left(q_{\mathrm{II}}+q_{\mathrm{II}}^{-1}\right)+43\left(q_{\mathrm{II}}^{2}+q_{\mathrm{II}}^{-2}\right)+8\left(q_{\mathrm{II}}^{3}+q_{\mathrm{II}}^{-3}\right) \\
& +\left(q_{\mathrm{I}}^{2}+q_{\mathrm{I}}^{-2}\right)\left(84+64\left(q_{\mathrm{II}}+q_{\mathrm{II}}^{-1}\right)+28\left(q_{\mathrm{II}}^{2}+q_{\mathrm{II}}^{-2}\right)+6\left(q_{\mathrm{II}}^{3}+q_{\mathrm{II}}^{-3}\right)\right) \\
& +\left(q_{\mathrm{I}}^{4}+q_{\mathrm{I}}^{-4}\right)\left(13+8\left(q_{\mathrm{II}}+q_{\mathrm{II}}^{-1}\right)+3\left(q_{\mathrm{II}}^{2}+q_{\mathrm{II}}^{-2}\right)\right), \\
\chi_{\mathbf{1 3 8 6}}\left(q_{\mathrm{I}}, q_{\mathrm{II}}\right)= & 178+144\left(q_{\mathrm{II}}+q_{\mathrm{II}}^{-1}\right)+73\left(q_{\mathrm{II}}^{2}+q_{\mathrm{II}}^{-2}\right)+24\left(q_{\mathrm{II}}^{3}+q_{\mathrm{II}}^{-3}\right)+3\left(q_{\mathrm{II}}^{4}+q_{\mathrm{II}}^{-4}\right) \\
& +\left(q_{\mathrm{I}}^{2}+q_{\mathrm{I}}^{-2}\right)\left(88+66\left(q_{\mathrm{II}}+q_{\mathrm{II}}^{-1}\right)+28\left(q_{\mathrm{II}}^{2}+q_{\mathrm{II}}^{-2}\right)+6\left(q_{\mathrm{II}}^{3}+q_{\mathrm{II}}^{-3}\right)\right) \\
& +\left(q_{\mathrm{I}}^{4}+q_{\mathrm{I}}^{-4}\right)\left(24+16\left(q_{\mathrm{II}}+q_{\mathrm{II}}^{-1}\right)+4\left(q_{\mathrm{II}}^{2}+q_{\mathrm{II}}^{-2}\right)\right)+\left(q_{\mathrm{I}}^{6}+q_{\mathrm{I}}^{-6}\right)\left(4+2\left(q_{\mathrm{II}}+q_{\mathrm{II}}^{-1}\right)\right),
\end{aligned}
$$

for the congruency class $d \equiv 0 \bmod 4$,

$$
\begin{aligned}
\chi_{\mathbf{1 0}}\left(q_{\mathrm{I}}, q_{\mathrm{II}}\right)= & 4+2\left(q_{\mathrm{II}}+q_{\mathrm{II}}^{-1}\right)+q_{\mathrm{I}}^{2}+q_{\mathrm{I}}^{-2}, \\
\chi_{\mathbf{1 2 0}}\left(q_{\mathrm{I}}, q_{\mathrm{II}}\right)= & 24+16\left(q_{\mathrm{II}}+q_{\mathrm{II}}^{-1}\right)+4\left(q_{\mathrm{II}}^{2}+q_{\mathrm{II}}^{-2}\right)+\left(q_{\mathrm{I}}^{2}+q_{\mathrm{I}}^{-2}\right)\left(10+8\left(q_{\mathrm{II}}+q_{\mathrm{II}}^{-1}\right)+q_{\mathrm{II}}^{2}+q_{\mathrm{II}}^{-2}\right), \\
\chi_{\mathbf{1 2 6}}\left(q_{\mathrm{I}}, q_{\mathrm{II}}\right)= & 20+14\left(q_{\mathrm{II}}+q_{\mathrm{II}}^{-1}\right)+4\left(q_{\mathrm{II}}^{2}+q_{\mathrm{II}}^{-2}\right)+\left(q_{\mathrm{I}}^{2}+q_{\mathrm{I}}^{-2}\right)\left(13+8\left(q_{\mathrm{II}}+q_{\mathrm{II}}^{-1}\right)+3\left(q_{\mathrm{II}}^{2}+q_{\mathrm{II}}^{-2}\right)\right), \\
\chi_{\mathbf{3 2 0}}\left(q_{\mathrm{I}}, q_{\mathrm{II}}\right)= & 56+42\left(q_{\mathrm{II}}+q_{\mathrm{II}}^{-1}\right)+16\left(q_{\mathrm{II}}^{2}+q_{\mathrm{II}}^{-2}\right)+2\left(q_{\mathrm{II}}^{3}+q_{\mathrm{II}}^{-3}\right) \\
& +\left(q_{\mathrm{I}}^{2}+q_{\mathrm{I}}^{-2}\right)\left(24+16\left(q_{\mathrm{II}}+q_{\mathrm{II}}^{-1}\right)+4\left(q_{\mathrm{II}}^{2}+q_{\mathrm{II}}^{-2}\right)\right)+\left(q_{\mathrm{I}}^{4}+q_{\mathrm{I}}^{-4}\right)\left(4+2\left(q_{\mathrm{II}}+q_{\mathrm{II}}^{-1}\right)\right),
\end{aligned}
$$


for the congruency class $d \equiv 2 \bmod 4$ and

$$
\begin{aligned}
\chi_{\mathbf{1 6}}\left(q_{\mathrm{I}}, q_{\mathrm{II}}\right)= & 2\left(q_{\mathrm{I}}+q_{\mathrm{I}}^{-1}\right)\left(q_{\mathrm{II}}^{\frac{1}{2}}+q_{\mathrm{II}}^{-\frac{1}{2}}\right), \\
\chi_{\mathbf{1 4 4}}\left(q_{\mathrm{I}}, q_{\mathrm{II}}\right)= & 2\left(q_{\mathrm{I}}+q_{\mathrm{I}}^{-1}\right)\left(q_{\mathrm{II}}^{\frac{1}{2}}+q_{\mathrm{II}}^{-\frac{1}{2}}\right)\left[3+q_{\mathrm{II}}+q_{\mathrm{II}}^{-1}+q_{\mathrm{I}}^{2}+q_{\mathrm{I}}^{-2}\right], \\
\chi_{\mathbf{5 6 0}}\left(q_{\mathrm{I}}, q_{\mathrm{II}}\right)= & 2\left(q_{\mathrm{I}}+q_{\mathrm{I}}^{-1}\right)\left(q_{\mathrm{II}}^{\frac{1}{2}}+q_{\mathrm{II}}^{-\frac{1}{2}}\right)\left[7+6\left(q_{\mathrm{II}}+q_{\mathrm{II}}^{-1}\right)+q_{\mathrm{II}}^{2}+q_{\mathrm{II}}^{-2}\right. \\
& \left.+\left(q_{\mathrm{I}}^{2}+q_{\mathrm{I}}^{-2}\right)\left(3+2\left(q_{\mathrm{II}}+q_{\mathrm{II}}^{-1}\right)\right)\right], \\
\chi_{\mathbf{7 2 0}}\left(q_{\mathrm{I}}, q_{\mathrm{II}}\right)= & 2\left(q_{\mathrm{I}}+q_{\mathrm{I}}^{-1}\right)\left(q_{\mathrm{II}}^{\frac{1}{2}}+q_{\mathrm{II}}^{-\frac{1}{2}}\right)\left[11+6\left(q_{\mathrm{II}}+q_{\mathrm{II}}^{-1}\right)+3\left(q_{\mathrm{II}}^{2}+q_{\mathrm{II}}^{-2}\right)\right. \\
& \left.+\left(q_{\mathrm{I}}^{2}+q_{\mathrm{I}}^{-2}\right)\left(3+2\left(q_{\mathrm{II}}+q_{\mathrm{II}}^{-1}\right)\right)+q_{\mathrm{I}}^{4}+q_{\mathrm{I}}^{-4}\right],
\end{aligned}
$$

for the congruency class $d \equiv 1,3 \bmod 4$.

\section{B Data for $(2,1,2,1)$ model and $E_{7}$}

In this appendix we summarize the data to relate the instanton coefficients of the $(2,1,2,1)$ model with the free energy of the topological string theory on the local $E_{7}$ del Pezzo geometry. In appendix B.1 we collect the instanton coefficients, while appendix B.2 provides the decompositions of the irreducible representations of $E_{7}$ to the subalgebra so(12) $\times \operatorname{su}(2)$ and the characters of the so(12) representations.

\section{B.1 Instanton coefficients for $(2,1,2,1)$ model}

In this appendix we list the first several instanton coefficients. For the worldsheet coefficients, following the main text, we express them by separating into the \pm parts. Note that, purely from the numerical results of the instanton effects up to degree $d$, we only obtain the difference $\delta_{d}^{+}(k)-\delta_{d}^{-}(k)$. The separation is obtained only after studying the instanton effects up to degree $2 d$ or taking care of the tables for the BPS indices of the local del Pezzo $E_{7}$ geometry in [37]. For $\delta_{d}^{+}(k)$ we obtain

$$
\begin{aligned}
\delta_{1}^{+}(k)= & \frac{32}{\left(2 \sin \frac{\pi}{k}\right)^{2}}, \\
\delta_{2}^{+}(k)= & -\frac{144}{\left(2 \sin \frac{\pi}{k}\right)^{2}}+3 \\
\delta_{3}^{+}(k)= & \frac{1632}{\left(2 \sin \frac{\pi}{k}\right)^{2}}-128 \\
\delta_{4}^{+}(k)= & -\frac{29248}{\left(2 \sin \frac{\pi}{k}\right)^{2}}+6157-460\left(2 \sin \frac{\pi}{k}\right)^{2}+7\left(2 \sin \frac{\pi}{k}\right)^{4}, \\
\delta_{5}^{+}(k)= & \frac{652160}{\left(2 \sin \frac{\pi}{k}\right)^{2}}-288576+59328\left(2 \sin \frac{\pi}{k}\right)^{2}-6336\left(2 \sin \frac{\pi}{k}\right)^{4}+288\left(2 \sin \frac{\pi}{k}\right)^{6}, \\
\delta_{6}^{+}(k)= & -\frac{16629168}{\left(2 \sin \frac{\pi}{k}\right)^{2}}+13073657-5292592\left(2 \sin \frac{\pi}{k}\right)^{2}+1338304\left(2 \sin \frac{\pi}{k}\right)^{4} \\
& -215992\left(2 \sin \frac{\pi}{k}\right)^{6}+20969\left(2 \sin \frac{\pi}{k}\right)^{8}-1020\left(2 \sin \frac{\pi}{k}\right)^{10}+13\left(2 \sin \frac{\pi}{k}\right)^{12},
\end{aligned}
$$


while for $\delta_{d}^{-}(k)$ we obtain

$$
\begin{aligned}
\delta_{1}^{-}(k)= & \frac{24}{\left(2 \sin \frac{\pi}{k}\right)^{2}}, \\
\delta_{2}^{-}(k)= & -\frac{128}{\left(2 \sin \frac{\pi}{k}\right)^{2}}, \\
\delta_{3}^{-}(k)= & \frac{1608}{\left(2 \sin \frac{\pi}{k}\right)^{2}}-96 \\
\delta_{4}^{-}(k)= & -\frac{29184}{\left(2 \sin \frac{\pi}{k}\right)^{2}}+5888-384\left(2 \sin \frac{\pi}{k}\right)^{2} \\
\delta_{5}^{-}(k)= & \frac{651680}{\left(2 \sin \frac{\pi}{k}\right)^{2}}-286320+57552\left(2 \sin \frac{\pi}{k}\right)^{2}-5776\left(2 \sin \frac{\pi}{k}\right)^{4}+216\left(2 \sin \frac{\pi}{k}\right)^{6}, \\
\delta_{6}^{-}(k)= & -\frac{16626048}{\left(2 \sin \frac{\pi}{k}\right)^{2}}+13053696-5262208\left(2 \sin \frac{\pi}{k}\right)^{2}+1316608\left(2 \sin \frac{\pi}{k}\right)^{4} \\
& -207232\left(2 \sin \frac{\pi}{k}\right)^{6}+18944\left(2 \sin \frac{\pi}{k}\right)^{8}-768\left(2 \sin \frac{\pi}{k}\right)^{10} \cdot
\end{aligned}
$$

For the membrane instanton, the derivation is more direct. We only need to apply $(6.7)$, rewrite into the multi-covering expression and separate into $\beta_{d}^{ \pm}(k)$ according to the arguments of the sine functions in the numerators. For $\beta_{d}^{+}(k)$ we obtain

$$
\begin{aligned}
\beta_{1}^{+}(k)= & -\frac{4 \sin 2 \pi k}{\pi \sin ^{2} \pi k}, \\
\beta_{2}^{+}(k)= & \frac{50 \sin 2 \pi k+11 \sin 4 \pi k}{2 \pi \sin ^{2} \pi k}, \\
\beta_{3}^{+}(k)= & -\frac{12(15 \sin 2 \pi k+15 \sin 4 \pi k+2 \sin 6 \pi k)}{\pi \sin ^{2} \pi k}, \\
\beta_{4}^{+}(k)= & \frac{4(863 \sin 2 \pi k+1630 \sin 4 \pi k+869 \sin 6 \pi k+138 \sin 8 \pi k+6 \sin 10 \pi k)}{2 \pi \sin ^{2} \pi k}, \\
\beta_{5}^{+}(k)= & -40(560 \sin 2 \pi k+1317 \sin 4 \pi k+1318 \sin 6 \pi k+576 \sin 8 \pi k \\
& +127 \sin 10 \pi k+16 \sin 12 \pi k+\sin 14 \pi k) /\left(\pi \sin { }^{2} \pi k\right), \\
\beta_{6}^{+}(k)= & 3(248502 \sin 2 \pi k+608220 \sin 4 \pi k+824190 \sin 6 \pi k+610860 \sin 8 \pi k \\
& +265298 \sin 10 \pi k+77619 \sin 12 \pi k+16796 \sin 14 \pi k+2652 \sin 16 \pi k+280 \sin 18 \pi k \\
& +12 \sin 20 \pi k) /\left(2 \pi \sin ^{2} \pi k\right),
\end{aligned}
$$

while for $\beta_{d}^{-}(k)$ we obtain

$$
\begin{aligned}
& \beta_{1}^{-}(k)=-\frac{9 \sin \pi k+\sin 3 \pi k}{2 \pi \sin ^{2} \pi k}, \\
& \beta_{2}^{-}(k)=\frac{16 \sin \pi k+16 \sin 3 \pi k}{\pi \sin ^{2} \pi k}, \\
& \beta_{3}^{-}(k)=-\frac{3(56 \sin \pi k+152 \sin 3 \pi k+57 \sin 5 \pi k+\sin 7 \pi k)}{2 \pi \sin ^{2} \pi k}, \\
& \beta_{4}^{-}(k)=\frac{4(368 \sin \pi k+1392 \sin 3 \pi k+1392 \sin 5 \pi k+400 \sin 7 \pi k+32 \sin 9 \pi k)}{2 \pi \sin ^{2} \pi k},
\end{aligned}
$$




$$
\begin{aligned}
\beta_{5}^{-}(k)= & -5(3888 \sin \pi k+15280 \sin 3 \pi k+23489 \sin 5 \pi k+15348 \sin 7 \pi k+4655 \sin 9 \pi k \\
& +767 \sin 11 \pi k+68 \sin 13 \pi k+\sin 15 \pi k) /\left(2 \pi \sin ^{2} \pi k\right), \\
\beta_{6}^{-}(k)= & 48(3503 \sin \pi k+13119 \sin 3 \pi k+23847 \sin 5 \pi k+23873 \sin 7 \pi k+13336 \sin 9 \pi k \\
& +4671 \sin 11 \pi k+1168 \sin 13 \pi k+217 \sin 15 \pi k+28 \sin 17 \pi k+2 \sin 19 \pi k) /\left(\pi \sin ^{2} \pi k\right) .
\end{aligned}
$$

\section{B.2 Decomposition of $E_{7}$ representations}

To identify the representations which the BPS indices consist of for the $(2,1,2,1)$ model, we need to decompose the $E_{7}$ representations to the subalgebra so(12) $\times \mathrm{su}(2)$ and further decompose the so(12) representations to the subalgebra $\mathrm{so}(10) \times \mathrm{u}(1)$. The first several decompositions are given in table A.88 of [51]. Though this is not enough we can continue by the Mathematica package provided there. For our purpose, we separate the decompositions by the congruency class. For the even congruency class we find the decompositions

$$
\begin{aligned}
& 1 \rightarrow(1,1), \\
& 133 \rightarrow(1,3)+(\overline{32}, 2)+(66,1), \\
& 1463 \rightarrow(66,1)+(77,3)+(\overline{352}, 2)+(462,1), \\
& \mathbf{1 5 3 9} \rightarrow(\mathbf{1}, \mathbf{1})+(\overline{\mathbf{3 2}}, \mathbf{2})+(\mathbf{6 6}, 3)+(\mathbf{7 7}, \mathbf{1})+(\overline{\mathbf{3 5 2}}, \mathbf{2})+(\mathbf{4 9 5}, \mathbf{1}), \\
& 7371 \rightarrow(1,1)+(1,5)+(\overline{32}, 2)+(\overline{32}, 4)+(66,3)+(\overline{462}, 3)+(495,1)+(1638,1) \\
& +(\overline{\mathbf{1 7 2 8}}, \mathbf{2}) \text {, } \\
& 8645 \rightarrow(\mathbf{1}, 3)+(\overline{32}, 2)+(\overline{32}, 4)+(66,1)+(66,3)+(\overline{352}, 2)+(\overline{462}, 1)+(495,3) \\
& +(\overline{\mathbf{1 7 2 8}}, 2)+(\mathbf{2 0 7 9}, \mathbf{1}) \text {, } \\
& 40755 \rightarrow(\overline{32}, 2)+(66,1)+(66,3)+(77,1)+(77,3)+2(\overline{352}, 2)+(\overline{352}, 4)+(462,3), \\
& +(495,1)+(495,3)+(\overline{\mathbf{1 7 2 8}}, 2)+(2079,1)+(2079,3)+\left(\overline{\mathbf{2 1 1 2}}_{2}, 2\right)+\left(\overline{4928}^{\prime}, 2\right) \\
& +(8085,1) \text {, }
\end{aligned}
$$

while for the odd congruency class we find the decompositions

$$
\begin{aligned}
& 56 \rightarrow(12,2)+(32,1), \\
& 912 \rightarrow(12,2)+(32,3)+(220,2)+(352,1), \\
& 6480 \rightarrow(12,2)+(12,4)+(32,1)+(32,3)+(220,2)+(352,1)+(352,3)+(560,2) \\
& +(792,2)+(1728,1), \\
& 24320 \rightarrow\left(352^{\prime}, 4\right)+(560,2)+(1728,1)+(2112,3)+(4224,1)+(4752,2), \\
& 27664 \rightarrow(12,2)+(32,1)+(32,3)+(220,2)+(220,4)+(352,1)+(352,3)+(560,2) \\
& +(792,2)+(1728,3)+(2112,1)+\left(4928^{\prime}, 1\right)+(4928,2), \\
& 51072 \rightarrow(12,2)+(32,1)+(220,2)+(352,1)+\left(352^{\prime}, 2\right)+(352,3)+(560,2)+(560,4) \\
& +(792,2)+(1728,1)+(1728,3)+(2112,1)+(2112,3)+(4752,2)+(4928,2) \\
& +(8800,1) \text {, } \\
& 86184 \rightarrow(12,2)+(12,4)+(32,1)+(32,3)+(32,5)+2(220,2)+(220,4)+(352,1) \\
& +2(\mathbf{3 5 2}, 3)+(\mathbf{5 6 0}, \mathbf{2})+(\mathbf{7 9 2}, \mathbf{2})+(\mathbf{7 9 2}, 4)+(\mathbf{1 7 2 8}, \mathbf{1})+(\mathbf{1 7 2 8 , 3})+(\overline{\mathbf{4 7 5 2}, 2}) \\
& +\left(4928^{\prime}, 1\right)+(4928,2)+\left(4928^{\prime}, 3\right)+(8008,2)+(13728,1) .
\end{aligned}
$$


For the study of the membrane instantons in the $(2,1,2,1)$ model, we need to further decompose the so(12) representations to the subalgebra so $(10) \times u(1)$. For this purpose, the characters are helpful. These characters can be obtained by choosing $\xi=$ (2 $\log q, 0,0,0,0,0)$ in the Weyl character formula (A.6) if we fix the fundamental weights as

$$
\begin{array}{ll}
\omega_{1}=(1,0,0,0,0,0), & \omega_{2}=(1,1,0,0,0,0), \quad \omega_{3}=(1,1,1,0,0,0), \quad \omega_{4}=(1,1,1,1,0,0), \\
\omega_{5}=\left(\frac{1}{2}, \frac{1}{2}, \frac{1}{2}, \frac{1}{2}, \frac{1}{2},-\frac{1}{2}\right), \quad \omega_{6}=\left(\frac{1}{2}, \frac{1}{2}, \frac{1}{2}, \frac{1}{2}, \frac{1}{2}, \frac{1}{2}\right) . & \text { (B.7) }
\end{array}
$$

The explicit form of the characters is given by

$$
\begin{aligned}
& \chi_{\mathbf{1}}(q)=1, \\
& \chi_{\mathbf{1 2}}(q)=10+\left(q^{2}+q^{-2}\right), \\
& \chi_{\mathbf{3 2}}(q)=16\left(q+q^{-1}\right), \\
& \chi_{\mathbf{6 6}}(q)=46+10\left(q^{2}+q^{-2}\right), \\
& \chi_{\mathbf{7 7}}(q)=55+10\left(q^{2}+q^{-2}\right)+\left(q^{4}+q^{-4}\right), \\
& \chi_{\mathbf{2 2 0}}(q)=130+45\left(q^{2}+q^{-2}\right), \\
& \chi_{\mathbf{3 5 2}}(q)=160\left(q+q^{-1}\right)+16\left(q^{3}+q^{-3}\right), \\
& \chi_{\mathbf{3 5 2}^{\prime}}(q)=220+55\left(q^{2}+q^{-2}\right)+10\left(q^{4}+q^{-4}\right)+\left(q^{6}+q^{-6}\right) \text {, } \\
& \chi_{\mathbf{4 6 2}}(q)=210+126\left(q^{2}+q^{-2}\right), \\
& \chi_{495}(q)=255+120\left(q^{2}+q^{-2}\right), \\
& \chi_{\mathbf{5 6 0}}(q)=340+100\left(q^{2}+q^{-2}\right)+10\left(q^{4}+q^{-4}\right), \\
& \chi_{\mathbf{7 9 2}}(q)=372+210\left(q^{2}+q^{-2}\right), \\
& \chi_{\mathbf{1 2 8 7}}(q)=715+220\left(q^{2}+q^{-2}\right)+55\left(q^{4}+q^{-4}\right)+10\left(q^{6}+q^{-6}\right)+\left(q^{8}+q^{-8}\right), \\
& \chi_{\mathbf{1 6 3 8}}(q)=870+330\left(q^{2}+q^{-2}\right)+54\left(q^{4}+q^{-4}\right), \\
& \chi_{\mathbf{1 7 2 8}}(q)=720\left(q+q^{-1}\right)+144\left(q^{3}+q^{-3}\right) \text {, } \\
& \chi_{\mathbf{2 0 7 9}}(q)=1089+450\left(q^{2}+q^{-2}\right)+45\left(q^{4}+q^{-4}\right), \\
& \chi_{\mathbf{2 1 1 2}}(q)=880\left(q+q^{-1}\right)+160\left(q^{3}+q^{-3}\right)+16\left(q^{5}+q^{-5}\right), \\
& \chi_{\mathbf{2 8 6 0}}(q)=1540+550\left(q^{2}+q^{-2}\right)+100\left(q^{4}+q^{-4}\right)+10\left(q^{6}+q^{-6}\right), \\
& \chi_{\mathbf{4 0 0 4}}(q)=2002+715\left(q^{2}+q^{-2}\right)+220\left(q^{4}+q^{-4}\right)+55\left(q^{6}+q^{-6}\right)+10\left(q^{8}+q^{-8}\right) \\
& +\left(q^{10}+q^{-10}\right) \text {, } \\
& \chi_{\mathbf{4 2 2 4}}(q)=1440\left(q+q^{-1}\right)+672\left(q^{3}+q^{-3}\right), \\
& \chi_{\mathbf{4 7 5 2}}(q)=1980+1260\left(q^{2}+q^{-2}\right)+126\left(q^{4}+q^{-4}\right), \\
& \chi_{4928}(q)=2288+1200\left(q^{2}+q^{-2}\right)+120\left(q^{4}+q^{-4}\right), \\
& \chi_{\mathbf{4 9 2 8}^{\prime}}(q)=1904\left(q+q^{-1}\right)+560\left(q^{3}+q^{-3}\right) \text {, } \\
& \chi_{\mathbf{8 0 0 8}}(q)=3740+1814\left(q^{2}+q^{-2}\right)+320\left(q^{4}+q^{-4}\right), \\
& \chi_{\mathbf{8 0 8 5}}(q)=3465+2100\left(q^{2}+q^{-2}\right)+210\left(q^{4}+q^{-4}\right), \\
& \chi_{\mathbf{8 8 0 0}}(q)=3200\left(q+q^{-1}\right)+1200\left(q^{3}+q^{-3}\right), \\
& \chi_{9152}(q)=3520\left(q+q^{-1}\right)+880\left(q^{3}+q^{-3}\right)+160\left(q^{5}+q^{-5}\right)+16\left(q^{7}+q^{-7}\right) \text {, }
\end{aligned}
$$




$$
\begin{aligned}
\chi_{\mathbf{9 5 0 4}}(q)= & 3312\left(q+q^{-1}\right)+1440\left(q^{3}+q^{-3}\right), \\
\chi_{\mathbf{1 1 0 1 1}}(q)= & 5005+2002\left(q^{2}+q^{-2}\right)+715\left(q^{4}+q^{-4}\right)+220\left(q^{6}+q^{-6}\right)+55\left(q^{8}+q^{-8}\right) \\
& +10\left(q^{10}+q^{-10}\right)+\left(q^{12}+q^{-12}\right), \\
\chi_{\mathbf{1 1 0 8 8}}(q)= & 5280+2310\left(q^{2}+q^{-2}\right)+540\left(q^{4}+q^{-4}\right)+54\left(q^{6}+q^{-6}\right), \\
\chi_{\mathbf{1 1 0 8 8 ^ { \prime }}}(q)= & 5368+2200\left(q^{2}+q^{-2}\right)+550\left(q^{4}+q^{-4}\right)+100\left(q^{6}+q^{-6}\right)+10\left(q^{8}+q^{-8}\right), \\
\chi_{\mathbf{1 1 2 3 2}}(q)= & 5292+2475\left(q^{2}+q^{-2}\right)+450\left(q^{4}+q^{-4}\right)+45\left(q^{6}+q^{-6}\right), \\
\chi_{\mathbf{1 3 7 2 8}}(q)= & 5280\left(q+q^{-1}\right)+1440\left(q^{3}+q^{-3}\right)+144\left(q^{5}+q^{-5}\right) .
\end{aligned}
$$

Open Access. This article is distributed under the terms of the Creative Commons Attribution License (CC-BY 4.0), which permits any use, distribution and reproduction in any medium, provided the original author(s) and source are credited.

\section{References}

[1] O. Aharony, O. Bergman, D.L. Jafferis and J. Maldacena, $N=6$ superconformal Chern-Simons-matter theories, M2-branes and their gravity duals, JHEP 10 (2008) 091 [arXiv:0806.1218] [INSPIRE].

[2] K. Hosomichi, K.-M. Lee, S. Lee, S. Lee and J. Park, $N=5,6$ superconformal Chern-Simons theories and M2-branes on orbifolds, JHEP 09 (2008) 002 [arXiv:0806.4977] [INSPIRE].

[3] O. Aharony, O. Bergman and D.L. Jafferis, Fractional M2-branes, JHEP 11 (2008) 043 [arXiv: 0807.4924] [INSPIRE].

[4] V. Pestun, Localization of gauge theory on a four-sphere and supersymmetric Wilson loops, Commun. Math. Phys. 313 (2012) 71 [arXiv:0712.2824] [InSPIRE].

[5] A. Kapustin, B. Willett and I. Yaakov, Exact results for Wilson loops in superconformal Chern-Simons theories with matter, JHEP 03 (2010) 089 [arXiv:0909.4559] [INSPIRE].

[6] Y. Hatsuda, S. Moriyama and K. Okuyama, Instanton effects in ABJM theory from Fermi gas approach, JHEP 01 (2013) 158 [arXiv:1211.1251] [INSPIRE].

[7] M. Mariño and P. Putrov, ABJM theory as a Fermi gas, J. Stat. Mech. 03 (2012) P03001 [arXiv: 1110.4066] [INSPIRE].

[8] Y. Hatsuda, M. Mariño, S. Moriyama and K. Okuyama, Non-perturbative effects and the refined topological string, JHEP 09 (2014) 168 [arXiv:1306.1734] [INSPIRE].

[9] N. Drukker, M. Mariño and P. Putrov, From weak to strong coupling in ABJM theory, Commun. Math. Phys. 306 (2011) 511 [arXiv:1007.3837] [INSPIRE].

[10] C.P. Herzog, I.R. Klebanov, S.S. Pufu and T. Tesileanu, Multi-matrix models and tri-Sasaki Einstein spaces, Phys. Rev. D 83 (2011) 046001 [arXiv: 1011.5487] [InSPIRE].

[11] N. Drukker, M. Mariño and P. Putrov, Nonperturbative aspects of ABJM theory, JHEP 11 (2011) 141 [arXiv:1103.4844] [INSPIRE].

[12] H. Fuji, S. Hirano and S. Moriyama, Summing up all genus free energy of ABJM matrix model, JHEP 08 (2011) 001 [arXiv: 1106.4631] [INSPIRE].

[13] Y. Hatsuda, S. Moriyama and K. Okuyama, Exact results on the ABJM Fermi gas, JHEP 10 (2012) 020 [arXiv:1207.4283] [INSPIRE]. 
[14] P. Putrov and M. Yamazaki, Exact ABJM partition function from TBA, Mod. Phys. Lett. A 27 (2012) 1250200 [arXiv:1207.5066] [INSPIRE].

[15] F. Calvo and M. Mariño, Membrane instantons from a semiclassical TBA, JHEP 05 (2013) 006 [arXiv: 1212.5118] [INSPIRE].

[16] Y. Hatsuda, S. Moriyama and K. Okuyama, Instanton bound states in ABJM theory, JHEP 05 (2013) 054 [arXiv: 1301.5184] [INSPIRE].

[17] A. Cagnazzo, D. Sorokin and L. Wulff, String instanton in $A d S_{4} \times C P^{3}$, JHEP 05 (2010) 009 [arXiv:0911.5228] [INSPIRE].

[18] Y. Hatsuda, S. Moriyama and K. Okuyama, Exact instanton expansion of the ABJM partition function, Prog. Theor. Exp. Phys. 2015 (2015) 11B104 [arXiv:1507.01678] [INSPIRE].

[19] M. Mariño, Localization at large- $N$ in Chern-Simons-matter theories, J. Phys. A 50 (2017) 443007 [arXiv: 1608.02959] [InSPIRE].

[20] A. Grassi, Y. Hatsuda and M. Mariño, Topological strings from quantum mechanics, Annales Henri Poincaré 17 (2016) 3177 [arXiv: 1410.3382] [INSPIRE].

[21] D. Gaiotto and E. Witten, Janus configurations, Chern-Simons couplings, and the theta-angle in $N=4$ super Yang-Mills theory, JHEP 06 (2010) 097 [arXiv:0804.2907] [INSPIRE].

[22] K. Hosomichi, K.-M. Lee, S. Lee, S. Lee and J. Park, $N=4$ superconformal Chern-Simons theories with hyper and twisted hyper multiplets, JHEP 07 (2008) 091 [arXiv:0805.3662] [INSPIRE].

[23] Y. Imamura and K. Kimura, On the moduli space of elliptic Maxwell-Chern-Simons theories, Prog. Theor. Phys. 120 (2008) 509 [arXiv:0806.3727] [INSPIRE].

[24] S. Terashima and F. Yagi, Orbifolding the membrane action, JHEP 12 (2008) 041 [arXiv:0807.0368] [INSPIRE].

[25] Y. Imamura and K. Kimura, $N=4$ Chern-Simons theories with auxiliary vector multiplets, JHEP 10 (2008) 040 [arXiv:0807.2144] [INSPIRE].

[26] S. Moriyama and T. Nosaka, Exact instanton expansion of superconformal Chern-Simons theories from topological strings, JHEP 05 (2015) 022 [arXiv: 1412.6243] [INSPIRE].

[27] Y. Hatsuda, M. Honda, S. Moriyama and K. Okuyama, ABJM Wilson loops in arbitrary representations, JHEP 10 (2013) 168 [arXiv:1306.4297] [INSPIRE].

[28] S. Matsumoto and S. Moriyama, ABJ fractional brane from ABJM Wilson loop, JHEP 03 (2014) 079 [arXiv:1310.8051] [INSPIRE].

[29] B. Assel, N. Drukker and J. Felix, Partition functions of $3 d \hat{D}$-quivers and their mirror duals from 1d free fermions, JHEP 08 (2015) 071 [arXiv:1504.07636] [INSPIRE].

[30] S. Moriyama and T. Nosaka, Superconformal Chern-Simons partition functions of affine D-type quiver from Fermi gas, JHEP 09 (2015) 054 [arXiv: 1504.07710] [INSPIRE].

[31] T. Nosaka, Instanton effects in ABJM theory with general R-charge assignments, JHEP 03 (2016) 059 [arXiv: 1512.02862] [INSPIRE].

[32] S. Moriyama and T. Suyama, Orthosymplectic Chern-Simons matrix model and chirality projection, JHEP 04 (2016) 132 [arXiv: 1601.03846] [INSPIRE]. 
[33] S. Moriyama and T. Nosaka, Orientifold ABJM matrix model: chiral projections and worldsheet instantons, JHEP 06 (2016) 068 [arXiv:1603.00615] [INSPIRE].

[34] S. Matsuno and S. Moriyama, Giambelli identity in super Chern-Simons matrix model, J. Math. Phys. 58 (2017) 032301 [arXiv:1603.04124] [INSPIRE].

[35] K. Kiyoshige and S. Moriyama, Dualities in ABJM matrix model from closed string viewpoint, JHEP 11 (2016) 096 [arXiv:1607.06414] [INSPIRE].

[36] S. Moriyama, S. Nakayama and T. Nosaka, Instanton effects in rank deformed superconformal Chern-Simons theories from topological strings, JHEP 08 (2017) 003 [arXiv: 1704.04358] [INSPIRE].

[37] M.-X. Huang, A. Klemm and M. Poretschkin, Refined stable pair invariants for E-, $M$ - and [p,q]-strings, JHEP 11 (2013) 112 [arXiv:1308.0619] [INSPIRE].

[38] A. Hanany and E. Witten, Type IIB superstrings, BPS monopoles and three-dimensional gauge dynamics, Nucl. Phys. B 492 (1997) 152 [hep-th/9611230] [INSPIRE].

[39] S. Moriyama and T. Nosaka, Partition functions of superconformal Chern-Simons theories from Fermi gas approach, JHEP 11 (2014) 164 [arXiv: 1407.4268] [INSPIRE].

[40] S.-S. Kim and F. Yagi, $5 d E_{n}$ Seiberg-Witten curve via toric-like diagram, JHEP 06 (2015) 082 [arXiv:1411.7903] [INSPIRE].

[41] Y. Hatsuda, M. Honda and K. Okuyama, Large- $N$ non-perturbative effects in $N=4$ superconformal Chern-Simons theories, JHEP 09 (2015) 046 [arXiv: 1505.07120] [INSPIRE].

[42] S. Moriyama and T. Nosaka, ABJM membrane instanton from a pole cancellation mechanism, Phys. Rev. D 92 (2015) 026003 [arXiv:1410.4918] [INSPIRE].

[43] S. Moriyama and T. Suyama, Instanton effects in orientifold ABJM theory, JHEP 03 (2016) 034 [arXiv: 1511.01660] [INSPIRE].

[44] M. Honda, Exact relations between M2-brane theories with and without orientifolds, JHEP 06 (2016) 123 [arXiv:1512.04335] [INSPIRE].

[45] K. Okuyama, Orientifolding of the ABJ Fermi gas, JHEP 03 (2016) 008 [arXiv: 1601.03215] [INSPIRE].

[46] F. Benini, S. Benvenuti and Y. Tachikawa, Webs of five-branes and $N=2$ superconformal field theories, JHEP 09 (2009) 052 [arXiv:0906.0359] [INSPIRE].

[47] M. Honda and S. Moriyama, Instanton effects in orbifold ABJM theory, JHEP 08 (2014) 091 [arXiv: 1404.0676] [INSPIRE].

[48] N. Seiberg, Five-dimensional SUSY field theories, nontrivial fixed points and string dynamics, Phys. Lett. B 388 (1996) 753 [hep-th/9608111] [INSPIRE].

[49] S. Codesido, A. Grassi and M. Mariño, Spectral theory and mirror curves of higher genus, Annales Henri Poincaré 18 (2017) 559 [arXiv: 1507.02096] [INSPIRE].

[50] S. Codesido, J. Gu and M. Mariño, Operators and higher genus mirror curves, JHEP 02 (2017) 092 [arXiv:1609.00708] [INSPIRE].

[51] R. Feger and T.W. Kephart, LieART - a Mathematica application for Lie algebras and representation theory, Comput. Phys. Commun. 192 (2015) 166 [arXiv:1206.6379] [INSPIRE]. 